Supporting Information for

\title{
Synthesis of Homoallylic Alcohols via Palladium-Catalyzed Three- Component Coupling of an Arylboronic Acid with Allenes and
}

\author{
Aldehydes \\ Chad D. Hopkins and Helena C. Malinakova* \\ Department of Chemistry, University of Kansas, 1251 Wescoe Hall Drive, \\ Lawrence, KS 66045
}

$\underline{\text { hmalina@ku.edu }}$

Table of Contents

\section{General Experimental}

Synthesis and Complete Characterization Data for Compounds 5-10 and 12.

References

Copies of ${ }^{1} \mathrm{H}$ NMR and ${ }^{13} \mathrm{C}$ NMR Spectra of Homoallylic alcohols 5-10 and Lactone 12.
Page

S-2 - S-9

S-10

S-11 - S-30 


\section{General Experimental}

Unless otherwise indicated, all NMR data were collected at room temperature in $\mathrm{CDCl}_{3}$ with internal $\mathrm{CHCl}_{3}$ as the reference $\left(\square 7.26 \mathrm{ppm}\right.$ for ${ }^{1} \mathrm{H}$ and $77.00 \mathrm{ppm}$ for $\left.{ }^{13} \mathrm{C}\right)$. Specific rotation was measured at room temperature in methylene chloride. IR spectra were measured in $\mathrm{KBr}$ pellets, or as thin films on salt $(\mathrm{NaCl})$ plates. Melting points are uncorrected and were taken in open capillary tubes. MS were measured under an electrospray (ES) conditions. Analytical thin-layer chromatography (TLC) was carried out on commercial Merck silica gel 60 plates, $250 \square \mathrm{m}$ thickness, with fluorescent indicator (F254) or stained with aqueous $\mathrm{KMnO}_{4}$ solution. Column chromatography was performed with 40-63 $\square \mathrm{m}$ silica gel (Merck). Preparative thin-layer chromatography was performed with $1000 \square \mathrm{m}$ commercial silica GF plates with fluorescent indicator (UV 254), Analtech. Tetrahydrofuran (THF) was freshly distilled from sodium/benzophenone. Diethyl ether was freshly distilled from $\mathrm{CaH}_{2}$. Methanol, toluene, 1,2-dichloroethane and DMF were kept over $3 \AA$ (8-12 mesh) molecular sieves under an atmosphere of dry argon; other solvents were used as received. Unless otherwise specified, all reactions were carried out under an atmosphere of argon in oven-dried (at least $6 \mathrm{~h}$ at $140{ }^{\circ} \mathrm{C}$ ) glassware. The arylboronic acid 2b $\left(p-\mathrm{MeOC}_{6} \mathrm{H}_{4} \mathrm{~B}(\mathrm{OH})_{2}\right)$ was purchased from Aldrich, purified by recrystallization from water, and dried under vacuum for at least 16 h. 4-Methoxyphenyltrimethyltin $2 \mathbf{a a}^{1} \quad 1,2-$ nonadiene $\mathbf{3}^{2}$ and (+)-(3,2,10- $\square$-pinene)palladium(II) chloride $\mathbf{1} \mathbf{b}^{3}$ were prepared according to the indicated literature protocols. ${ }^{1-3}$ Other materials were used as received from commercial suppliers.

Preparation of alcohol 5a via Conditions A. To the solid allylpalladium(II) dimer catalyst $1 \mathbf{a}(0.002 \mathrm{~g}, 0.005 \mathrm{mmol}, 0.05$ equiv) and triphenylphosphine $(0.003 \mathrm{~g}, 0.011$ mmol, 0.1 equiv) in the reaction vessel was injected a solution of 1,2-nonadiene $3(0.027 \mathrm{~g}$, $0.218 \mathrm{mmol}, 2.0$ equiv) and 4-methoxyphenyltrimethyltin $\mathbf{2 a}(0.030 \mathrm{~g}, 0.111 \mathrm{mmol}, 1.0$ 
equiv) in 1,2-dichloroethane (4.0 mL), followed by neat benzaldehyde $4 \mathbf{a}(0.012 \mathrm{~g}, 0.113$ mmol, $11 \square \mathrm{L}, 1.0$ equiv). The reaction mixture was heated to $60{ }^{\circ} \mathrm{C}$ under argon for $24 \mathrm{~h}$. The reaction mixture was filtered through a short plug of silica eluting with methylene chloride, and the crude product was separated by flash chromatography over silica eluting with EtOAc/hexane $(1: 10)$ to afford alcohol 5a $(0.014 \mathrm{~g}, 38 \%)$ as a pale yellow oil. Analytical data for alcohol 5a are provided below.

Preparation of alcohol 5 via Conditions B. To the solid allylpalladium(II) dimer catalyst $1 \mathrm{a}(0.008 \mathrm{~g}, 0.023 \mathrm{mmol}, 0.1 \mathrm{equiv})$ and tricyclohexylphosphine $(0.025 \mathrm{~g}, 0.090 \mathrm{mmol}, 0.4$ equiv), $p$-methoxyphenylboronic acid $2 \mathbf{b}(0.069 \mathrm{~g}, 0.451 \mathrm{mmol}, 2.0$ equiv) and $\mathrm{CsF}(0.068$ $\mathrm{g}, 0.452 \mathrm{mmol}, 2.0$ equiv) in the reaction vessel was injected a solution of 1,2-nonadiene 3 (0.056 g, $0.452 \mathrm{mmol}, 2.0$ equiv) in DMF $(3.0 \mathrm{~mL})$, followed by neat benzaldehyde $4 \mathbf{a}$ (0.024 g, $0.226 \mathrm{mmol}, 23 \square \mathrm{L}, 1.0$ equiv). The reaction mixture was stirred at $\mathrm{rt}$ under argon for $24 \mathrm{~h}$. Water $(20 \mathrm{~mL})$ was added, and the mixture was extracted with ether $(4 \mathrm{x} 20 \mathrm{~mL})$. Organic extracts were dried $\left(\mathrm{MgSO}_{4}\right)$, and the solvents were removed under reduced pressure to afford crude material that was purified by flash chromatography over silica eluting with EtOAc/hexane (1: 10), and preparative thin layer chromatography eluting with EtOAc/hexane (1 : 10) to afford pure separated diastereomers 5a $(0.041 \mathrm{~g}, 54 \%)$ and $\mathbf{5 b}$ (0.008 $\mathrm{g}, 10 \%)$ as colorless oils (64\% combined yield, $69 \%$ de).

General protocol for the preparation of homoallylic alcohols 5-10 via Conditions C. A solution of 1,2-nonadiene $3(0.140 \mathrm{~g}, 1.13 \mathrm{mmol}, 5.0$ equiv) in THF (3.0 mL) and the neat aldehyde $(0.226 \mathrm{mmol}, 1.0$ equiv) were sequentially injected into a reaction vessel containing the solid reagents including $p$-methoxyphenylboronic acid $\mathbf{2 b}(0.069 \mathrm{~g}, 0.451$ mmol, 2.0 equiv), (+)-(3,2,10- $\square$-pinene)palladium(II) chloride $1 \mathbf{b}(0.006 \mathrm{~g}, 0.011 \mathrm{mmol}$. 0.05 equiv), $\left[\mathrm{HPPh}(t-\mathrm{Bu})_{2}\right] \mathrm{BF}_{4}(0.007 \mathrm{~g}, 0.023 \mathrm{mmol}, 0.1$ equiv) and $\mathrm{CsF}(0.136 \mathrm{~g}, 0.903$ mmol, 4.0 equiv). The reaction mixture was stirred at $\mathrm{rt}$ under argon for $24 \mathrm{~h}$. Water (20 $\mathrm{mL})$ was added, and the mixture was extracted with ether (4 x $20 \mathrm{~mL})$. Organic extracts were dried $\left(\mathrm{MgSO}_{4}\right)$, and the solvents were removed under reduced pressure to afford crude 
products that were separated by flash chromatography over silica. Subsequent separation of diastereomers of alcohols 5-10 via preparative thin layer chromatography over silica afforded pure alcohols 5-10 as colorless to yellow oils.

( \pm$)-(1 R, 2 S)-2-(1-H e x y l)-3-(p-m e t h o x y p h e n y l)-1-p h e n y l-3-b u t e n-1-o l \quad(5 a)$ and $( \pm)-(1 S, 2 S)-2-(1-H e x y l)-3-(p-m e t h o x y p h e n y l)-1-p h e n y l-3-b u t e n-1-o l \quad(5 b)$. Treatment of benzaldehyde $4 \mathbf{a}(0.024 \mathrm{~g}, 0.226 \mathrm{mmol}, 23 \square \mathrm{L}, 1.0$ equiv) according to the general procedure described above including the exact quantities of reagents, followed by flash chromatography over silica eluting with EtOAc/hexane $(1: 10)$, and preparative TLC eluting with EtOAc/hexane (1 : 10) afforded pure separated diastereomers 5a $(0.049 \mathrm{~g}$, $64 \%)$ and $\mathbf{5 b}(0.009 \mathrm{~g}, 12 \%)$ as colorless oils (76\% combined yield, $67 \% \mathrm{de})$.

Analytical data for diastereomer 5a: $\mathrm{R}_{f}=0.38$ (EtOAc/hexane $\left.1: 5\right) ;{ }^{1} \mathrm{H}$ NMR (500 $\left.\mathrm{MHz}, \mathrm{CDCl}_{3}\right) \square 7.27-7.26(\mathrm{~m}, 4 \mathrm{H}), 7.21-7.18(\mathrm{~m}, 1 \mathrm{H}), 7.19(\mathrm{~d}, J=8.6 \mathrm{~Hz}, 2 \mathrm{H}), 6.82(\mathrm{~d}, J$ $=8.7 \mathrm{~Hz}, 2 \mathrm{H}), 5.34(\mathrm{~s}, 1 \mathrm{H}), 5.06(\mathrm{~s}, 1 \mathrm{H}), 4.65(\mathrm{dd}, J=4.8 \mathrm{~Hz}, 2.6 \mathrm{~Hz}, 1 \mathrm{H}), 3.80(\mathrm{~s}, 3 \mathrm{H})$, $2.93(\mathrm{dd}, J=14.1 \mathrm{~Hz}, 5.5 \mathrm{~Hz}, 1 \mathrm{H}), 1.97$ (d, $J=2.6 \mathrm{~Hz}, 1 \mathrm{H}), 1.67-1.58$ (m, $2 \mathrm{H}), 1.44-1.35$ $(\mathrm{m}, 1 \mathrm{H}), 1.23-1.10(\mathrm{~m}, 7 \mathrm{H}), 0.83(\mathrm{t}, J=7.1 \mathrm{~Hz}, 3 \mathrm{H}) ;{ }^{13} \mathrm{C} \mathrm{NMR}\left(125 \mathrm{MHz}, \mathrm{CDCl}_{3}\right)$ 159.2, 149.4, 143.0, 135.9, 128.2 (2 carbons), 127.9 (2 carbons), 127.2, 126.5 (2 carbons), 113.8 ( 2 carbons), 113.1, 75.8, 55.5, 52.1, 31.9, 29.7, 27.9, 27.6, 22.8, 14.3; IR (neat, $\mathrm{cm}^{-1}$ ) 3596 (w br), 1607 (m), 747 (m), 732 (s); HRMS (ES ${ }^{+}$) calcd for $\mathrm{C}_{23} \mathrm{H}_{34} \mathrm{NO}_{2}\left(\mathrm{M}+\mathrm{NH}_{4}^{+}\right)$, 356.2590, found 356.2602.

Analytical data for diastereomer 5b: $\mathrm{R}_{f}=0.44($ EtOAc/hexane $1: 3) ;{ }^{1} \mathrm{H}$ NMR (400 $\left.\mathrm{MHz}, \mathrm{CDCl}_{3}\right) \square 7.38-7.31(\mathrm{~m}, 6 \mathrm{H}), 7.29-7.27(\mathrm{~m}, 1 \mathrm{H}), 6.88(\mathrm{~d}, J=8.8 \mathrm{~Hz}, 2 \mathrm{H}), 5.42$ (s, 1 H), $5.19(\mathrm{~s}, 1 \mathrm{H}), 4.59$ (dd, $J=8.9 \mathrm{~Hz}, 2.4 \mathrm{~Hz}, 1 \mathrm{H}), 3.82(\mathrm{~s}, 3 \mathrm{H}), 2.86-2.81$ (m, $1 \mathrm{H}), 2.26$ $(\mathrm{d}, J=2.5 \mathrm{~Hz}, 1 \mathrm{H}), 1.29-1.15(\mathrm{~m}, 4 \mathrm{H}), 1.13-1.05(\mathrm{~m}, 4 \mathrm{H}), 0.93-0.83$ (m, $2 \mathrm{H}), 0.78$ (t, $J=$ 7.5 Hz, $3 \mathrm{H}) ;{ }^{13} \mathrm{C}$ NMR (125 MHz, $\left.\mathrm{CDCl}_{3}\right) \square 159.0,149.9,142.7,135.5,128.2$ (2 carbons), 128.0 ( 2 carbons), 127.7, 127.1 ( 2 carbons), 113.9, 113.6 ( 2 carbons), 77.7, 55.3, 53.5, 31.6, 31.4, 29.2, 27.0, 22.5, 14.0; IR (neat, $\mathrm{cm}^{-1}$ ) 3440 (w m), 1607 (w), 740 (s), 704 (s); HRMS $\left(\mathrm{ES}^{+}\right)$calcd for $\mathrm{C}_{23} \mathrm{H}_{31} \mathrm{O}_{2}\left(\mathrm{M}+\mathrm{H}^{+}\right), 339.2324$, found 339.2320. 


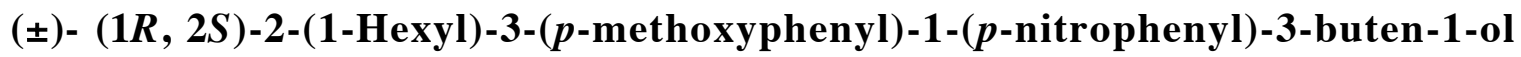
(6a) and ( \pm )- (1S, 2S)-2-(1-Hexyl)-3-(p-methoxyphenyl)-1-(p-nitrophenyl)-3buten-1-ol (6b). Treatment of $p$-nitrobenzaldehyde $4 \mathbf{b}(0.034 \mathrm{~g}, 0.226 \mathrm{mmol}, 1.0$ equiv) according to the general procedure described above including the exact quantities of reagents, followed by flash chromatography over silica eluting with EtOAc/hexane (1 : 5), and preparative TLC eluting with EtOAc/hexane (1 : 5) afforded pure separated diastereomers 6a $(0.049 \mathrm{~g}, 57 \%)$ and $\mathbf{6 b}(0.025 \mathrm{~g}, 28 \%)$ as yellow oils (85\% combined yield, $33 \%$ de).

Analytical data for diastereomer 6a: $\mathrm{R}_{f}=0.45$ (EtOAc/hexane $\left.1: 3\right) ;{ }^{1} \mathrm{H}$ NMR (500 $\left.\mathrm{MHz}, \mathrm{CDCl}_{3}\right) \square 8.16(\mathrm{~d}, J=8.8 \mathrm{~Hz}, 2 \mathrm{H}), 7.46(\mathrm{~d}, J=8.5 \mathrm{~Hz}, 2 \mathrm{H}), 7.21(\mathrm{dd}, J=6.7 \mathrm{~Hz}$, $2.0 \mathrm{~Hz}, 2 \mathrm{H}), 6.85(\mathrm{dd}, J=6.7 \mathrm{~Hz}, 2.0 \mathrm{~Hz}, 2 \mathrm{H}), 5.44(\mathrm{~s}, 1 \mathrm{H}), 5.11(\mathrm{~s}, 1 \mathrm{H}), 4.73$ (dd, $J=$ $4.2 \mathrm{~Hz}, 2.6 \mathrm{~Hz}, 1 \mathrm{H}), 3.83$ (s, $3 \mathrm{H}), 2.96(\mathrm{dt}, J=11.5 \mathrm{~Hz}, 3.7 \mathrm{~Hz}, 1 \mathrm{H}), 2.19$ (d, $J=2.6 \mathrm{~Hz}$, $1 \mathrm{H}), 1.75-1.60(\mathrm{~m}, 1 \mathrm{H}), 1.49-1.35(\mathrm{~m}, 2 \mathrm{H}), 1.27-1.17(\mathrm{~m}, 7 \mathrm{H}), 0.83(\mathrm{t}, J=7.1 \mathrm{~Hz}, 3 \mathrm{H})$; ${ }^{13} \mathrm{C}$ NMR (125 MHz, $\mathrm{CDCl}_{3}$ ) $\square 159.3,150.2,148.4,146.9,134.9,127.6$ (2 carbons), 127.1 (2 carbons), 123.2 (2 carbons), 113.8 (2 carbons), 112.2, 74.6, 55.3, 51.8, 31.6, 29.4, 27.3, 27.1, 22.5, 14.0; IR (neat, $\mathrm{cm}^{-1}$ ) 3550 (m br), 1606 (m), 1513 (s), 1346 (s), 853 (w), 825 (s), $705(\mathrm{~m})$; HRMS $\left(\mathrm{ES}^{+}\right)$calcd for $\mathrm{C}_{23} \mathrm{H}_{33} \mathrm{~N}_{2} \mathrm{O}_{4}\left(\mathrm{M}+\mathrm{NH}_{4}^{+}\right)$, 401.2440, found 401.2428.

Analytical data for diastereomer 6b: $\mathrm{R}_{f}=0.42($ EtOAc/hexane $1: 3) ;{ }^{1} \mathrm{H}$ NMR (500 $\left.\mathrm{MHz} \mathrm{CDCl}_{3}\right) \square 8.15(\mathrm{~d}, J=6.9 \mathrm{~Hz}, 2 \mathrm{H}), 7.49(\mathrm{~d}, J=8.7 \mathrm{~Hz}, 2 \mathrm{H}), 7.26(\mathrm{~d}, J=8.2 \mathrm{~Hz}, 2$ H), $6.84(\mathrm{~d}, J=6.7 \mathrm{~Hz}, 2 \mathrm{H}), 5.44(\mathrm{~s}, 1 \mathrm{H}), 5.16(\mathrm{~s}, 1 \mathrm{H}), 4.73(\mathrm{dd}, J=7.8 \mathrm{~Hz}, 3.2 \mathrm{~Hz}, 1 \mathrm{H})$, 2.88-2.84 (m, $1 \mathrm{H}), 2.41$ (d, J = 3.2 Hz, $1 \mathrm{H}), 1.47-1.37$ (m, $1 \mathrm{H}), 1.29-1.20$ (m, $2 \mathrm{H}), 1.19$ $1.26(\mathrm{~m}, 3 \mathrm{H}), 1.11-1.00(\mathrm{~m}, 4 \mathrm{H}), 0.80(\mathrm{t}, J=7.2 \mathrm{~Hz}, 3 \mathrm{H}) ;{ }^{13} \mathrm{C}$ NMR (125 MHz, $\left.\mathrm{CDCl}_{3}\right)$ 159.2, 150.1, 148.6, 147.4, 134.9, 127.9 (2 carbons), 127.7 (2 carbons) 123.3 (2 carbons), 114.7, 113.7 (2 carbons), 76.2, 55.3, 53.3, 31.6, 31.1, 29.2, 27.1, 22.5, 13.9; IR (neat, $\mathrm{cm}^{-1}$ ) 3518 (m br), $1606(\mathrm{~s}), 1513(\mathrm{~s}), 1346$ (s), 856 (m), 836 (m), 702 (m); HRMS (ES $\left.{ }^{+}\right)$calcd for $\mathrm{C}_{23} \mathrm{H}_{33} \mathrm{~N}_{2} \mathrm{O}_{4}\left(\mathrm{M}+\mathrm{NH}_{4}^{+}\right), 401.2440$, found 401.2433. 
( \pm - (1R, 2S)-2-(1-Hexyl)-3-(p-methoxyphenyl)-1-(3-pyridyl)-3-buten-1-ol (7a)

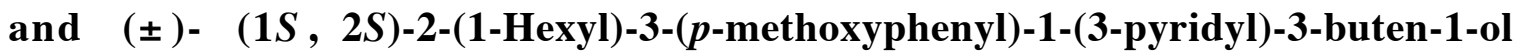
(7b). Treatment of 3-pyridinecarboxaldehyde 4c (0.024 g, 0.226 mmol, $21 \square \mathrm{L}, 1.0$ equiv) according to the general procedure described above including the exact quantities of reagents, followed by flash chromatography over silica eluting with EtOAc/hexane (3:1), and preparative TLC eluting with EtOAc/hexane $(3: 1)$ afforded an inseparable mixture of diastereomers $7 \mathbf{a}$ and $7 \mathbf{b}\left(0.054 \mathrm{~g}, 71 \%, \mathbf{7 a}: 7 \mathbf{b}=8.1: 1,78 \%\right.$ de by $\left.{ }^{1} \mathrm{H} \mathrm{NMR}\right)$ as a yellow oil.

Analytical data for the mixture of diastereomers $7 \mathbf{a}: 7 \mathbf{b}=\mathbf{8 . 1}: \mathbf{1}: \mathbf{R}_{f}=0.37$ (EtOAc/hexane 6 : 1); ${ }^{1} \mathrm{H}$ NMR (500 MHz, $\left.\mathrm{CDCl}_{3}\right) \square 8.60-8.56$ (m, $\left.0.11 \mathrm{H}\right), 8.47$ (s, br, 1 H), $8.42(\mathrm{~d}, J=4.7 \mathrm{~Hz}, 0.89 \mathrm{H}), 7.70(\mathrm{~d}, J=7.9 \mathrm{~Hz}, 0.11 \mathrm{H}), 7.69(\mathrm{~d}, J=7.8 \mathrm{~Hz}, 0.89 \mathrm{H})$, $7.32(\mathrm{~d}, J=8.8 \mathrm{~Hz}, 0.11 \mathrm{H}), 7.16(\mathrm{dd}, J=8.8 \mathrm{~Hz}, 2.2 \mathrm{~Hz}, 2.89 \mathrm{H}), 6.87(\mathrm{dd}, J=8.7 \mathrm{~Hz}$, $0.22 \mathrm{H}), 6.82(\mathrm{dd}, J=8.7 \mathrm{~Hz}, 1.78 \mathrm{H}), 5.44(\mathrm{~s}, 0.11 \mathrm{H}), 5.36(\mathrm{~s}, 0.89 \mathrm{H}), 5.16(\mathrm{~s}, 0.11 \mathrm{H})$, $5.06(\mathrm{~s}, 0.89 \mathrm{H}), 4.99(\mathrm{dt}, J=9.8 \mathrm{~Hz}, 0.9 \mathrm{~Hz}, 0.11 \mathrm{H}), 4.65(\mathrm{~d}, J=5.2 \mathrm{~Hz}, 0.89 \mathrm{H}), 3.82$ (s, $0.33 \mathrm{H}), 3.80(\mathrm{~s}, 2.67 \mathrm{H}), 2.92(\mathrm{q}, \mathrm{br}, J=6.4 \mathrm{~Hz}, 1 \mathrm{H}), 2.51(\mathrm{~d}, J=2.4 \mathrm{~Hz}, 0.11 \mathrm{H}), 2.31$ (d, $J=2.0 \mathrm{~Hz}, 0.89 \mathrm{H}), 1.69-1.62(\mathrm{~m}, 2 \mathrm{H}), 1.43-1.34(\mathrm{~m}, 1 \mathrm{H}), 1.27-1.09$ (m, $7 \mathrm{H}), 0.84(\mathrm{t}, J=$ $7.1 \mathrm{~Hz}, 2.67 \mathrm{H}), 0.78(\mathrm{t}, J=7.2 \mathrm{~Hz}, 0.33 \mathrm{H}) ;{ }^{13} \mathrm{C} \mathrm{NMR}\left(125 \mathrm{MHz}, \mathrm{CDCl}_{3}\right) \square(159.1)$, 159.1, (149.0), (148.8), 148.6, 148.4, 148.3, 138.1, (137.9), 135.3, (135.0), (134.4), 133.9, (127.9), 127.6 (2 carbons), (123.2), 122.9, (114.4), 113.7 (2 carbons), 113.2, (112.0), (75.1), 73.8, 55.3, (53.3), (52.1), 51.8, 31.7, (31.6), (31.0), 29.5, (29.2), 28.1, 27.3, (27.0), 22.6, (22.5), 14.0, (13.9) signals for the minor diastereomer are given in parentheses; IR (neat, $\mathrm{cm}^{-1}$ ) 3206 (w s), 1607 (s), 1577 (s), 895 (m), 836 (m), 808 (m), 715 (m); HRMS $\left(\mathrm{ES}^{+}\right)$calcd for $\mathrm{C}_{22} \mathrm{H}_{30} \mathrm{NO}_{2}\left(\mathrm{M}+\mathrm{H}^{+}\right), 340.2277$, found 340.2264.

( \pm )- (1R, 1S)-1-(2-Furyl)-2-(1-hexyl)-3-(p-methoxyphenyl)-3-buten-1-ol (8a) and ( \pm )- (1S, 1S)-1-(2-Furyl)-2-(1-hexyl)-3-( $p$-methoxyphenyl)-3-buten-1-ol (8b). Treatment of 2-furaldehyde $4 \mathbf{d}(0.022 \mathrm{~g}, 0.226 \mathrm{mmol}, 19 \square \mathrm{L}, 1.0$ equiv) according to the general procedure described above including the exact quantities of reagents, followed by 
flash chromatography over silica eluting with EtOAc/hexane (1: 7), and preparative TLC eluting with EtOAc/hexane (1 : 7) afforded pure separated diastereomers 8a $(0.047 \mathrm{~g}, 63 \%)$ and $\mathbf{8 b}(0.010 \mathrm{~g}, 13 \%)$ as colorless oils (76\% combined yield, $64 \% \mathrm{de})$.

Analytical data for diastereomer 8a: $\mathrm{R}_{f}=0.51($ EtOAc/hexane $1: 3) ;{ }^{1} \mathrm{H}$ NMR (500 $\left.\mathrm{MHz}_{\mathrm{CDCl}}\right) \square 7.28(\mathrm{t}, J=1.0 \mathrm{~Hz}, 1 \mathrm{H}), 7.22(\mathrm{~d}, J=8.7 \mathrm{~Hz}, 2 \mathrm{H}), 6.81(\mathrm{~d}, J=8.8 \mathrm{~Hz}, 2$ H), $6.26(\mathrm{dd}, J=3.2 \mathrm{~Hz}, 1.8 \mathrm{~Hz}, 1 \mathrm{H}), 6.16(\mathrm{~d}, J=3.2 \mathrm{~Hz}, 1 \mathrm{H}), 5.31(\mathrm{~s}, 1 \mathrm{H}), 5.03$ (s, $1 \mathrm{H})$, $4.65(\mathrm{t}, J=5.5 \mathrm{~Hz}, 1 \mathrm{H}), 3.89$ (s, $3 \mathrm{H}), 3.11-3.08(\mathrm{~m}, 1 \mathrm{H}), 1.96(\mathrm{~d}, J=4.9 \mathrm{~Hz}, 1 \mathrm{H}), 1.79$ $1.73(\mathrm{~m}, 1 \mathrm{H}), 1.66-1.58(\mathrm{~m}, 1 \mathrm{H}), 1.43-1.37(\mathrm{~m}, 1 \mathrm{H}), 1.25-1.22(\mathrm{~m}, 7 \mathrm{H}), 0.85(\mathrm{t}, J=7.0$ $\mathrm{Hz}, 3 \mathrm{H}) ;{ }^{13} \mathrm{C}$ NMR $\left(125 \mathrm{MHz}, \mathrm{CDCl}_{3}\right) \square 158.9,155.4,148.6,141.4,135.3,127.7$ (2 carbons), 113.6 (2 carbons), 112.9, 110.1, 106.5, 70.8, 55.2, 49.2, 31.7, 29.5, 29.2, 27.2, 22.6, 14.1; IR (neat, $\mathrm{cm}^{-1}$ ) 3431 (w s), 1608 (m), 835 (m), 734 (m); HRMS (ES ${ }^{+}$) calcd for $\mathrm{C}_{21} \mathrm{H}_{32} \mathrm{NO}_{3}\left(\mathrm{M}+\mathrm{NH}_{4}^{+}\right), 346.2382$, found 346.2373.

Analytical data for diastereomer 8b: $\mathrm{R}_{f}=0.47($ EtOAc/hexane $1: 3) ;{ }^{1} \mathrm{H}$ NMR (500 $\left.\mathrm{MHz}_{\mathrm{CDCl}}\right) \square 7.35(\mathrm{~d}, J=1.0 \mathrm{~Hz}, 1 \mathrm{H}), 7.31(\mathrm{~d}, J=8.7 \mathrm{~Hz}, 2 \mathrm{H}), 6.85(\mathrm{~d}, J=8.7 \mathrm{~Hz}, 2$ H), $6.30(\mathrm{dd}, J=3.1 \mathrm{~Hz}, 1.8 \mathrm{~Hz}, 1 \mathrm{H}), 6.25(\mathrm{~d}, J=3.1 \mathrm{~Hz}, 1 \mathrm{H}), 5.39$ (s, $1 \mathrm{H}), 5.17$ (s, $1 \mathrm{H})$, $4.65(\mathrm{dd}, J=8.7 \mathrm{~Hz}, 4.2 \mathrm{~Hz}, 1 \mathrm{H}), 3.81(\mathrm{~s}, 3 \mathrm{H}), 3.08$ (td, $J=8.5 \mathrm{~Hz}, 3.2 \mathrm{~Hz}, 1 \mathrm{H}), 2.22$ (d, $J=4.3 \mathrm{~Hz}, 1 \mathrm{H}), 1.39-1.31(\mathrm{~m}, 1 \mathrm{H}), 1.29-1.25$ (m, $2 \mathrm{H}), 1.22-1.17$ (m, $3 \mathrm{H}), 1.17-1.13$ (m, $4 \mathrm{H}), 0.82(\mathrm{t}, J=7.2 \mathrm{~Hz}, 3 \mathrm{H}) ;{ }^{13} \mathrm{C}$ NMR $\left(125 \mathrm{MHz}, \mathrm{CDCl}_{3}\right) \square 159.0,154.9,149.4,141.9$, 135.0, 128.1 (2 carbons), 114.1, 113.6 (2 carbons), 110.1, 107.5, 70.7, 55.3, 50.7, 31.6, 31.4, 29.2, 26.9, 22.5, 14.0; IR (neat, $\mathrm{cm}^{-1}$ ) 3582 (br w), 1607 (m), 836 (w), 737 (s); HRMS $\left(\mathrm{ES}^{+}\right)$calcd for $\mathrm{C}_{21} \mathrm{H}_{32} \mathrm{NO}_{3}\left(\mathrm{M}+\mathrm{NH}_{4}^{+}\right), 346.2382$, found 346.2383.

(土)- (1R, 2S)-2-(1-Hexyl)-1,3-bis(p-methoxyphenyl)-3-buten-1-ol (9a). Treatment of 4-anisaldehyde $4 \mathrm{e}(0.031 \mathrm{~g}, 0.226 \mathrm{mmol}, 27 \square \mathrm{L}, 1.0$ equiv) according to the general procedure described above including the exact quantities of reagents, followed by flash chromatography over silica eluting with EtOAc/hexane (1:7), and preparative TLC eluting with EtOAc/hexane $(1: 7)$ afforded a pure diastereomer 9a $(0.046 \mathrm{~g}, 54 \%)$ as a yellow oil. The presence of traces $(<3 \%)$ of the minor diastereomer was detected by ${ }^{1} \mathrm{H}$ NMR in the 
crude reaction mixture, but the diastereomer could not be isolated in sufficient purity to permit a complete characterization.

Analytical data for diastereomer 9a: $\mathrm{R}_{f}=0.39($ EtOAc/hexane $1: 3) ;{ }^{1} \mathrm{H}$ NMR (500 $\left.\mathrm{MHz} \mathrm{CDCl}_{3}\right) \square 7.17(\mathrm{dd}, J=8.7 \mathrm{~Hz}, 3.4 \mathrm{~Hz}, 4 \mathrm{H}), 6.81(\mathrm{dd}, J=8.5 \mathrm{~Hz}, 6.0 \mathrm{~Hz}, 4 \mathrm{H}), 5.32$ (s, $1 \mathrm{H}), 5.03(\mathrm{~s}, 1 \mathrm{H}), 4.60(\mathrm{dd}, J=5.2 \mathrm{~Hz}, 2.3 \mathrm{~Hz}, 1 \mathrm{H}), 3.80(\mathrm{~s}, 3 \mathrm{H}), 3.77$ (s, $3 \mathrm{H}), 2.89$ (pent, $J=4.7 \mathrm{~Hz}, 1 \mathrm{H}), 1.93(\mathrm{~d}, J=2.5 \mathrm{~Hz}, 1 \mathrm{H}), 1.71-1.58(\mathrm{~m}, 2 \mathrm{H}), 1.46-1.38(\mathrm{~m}, 1 \mathrm{H})$, 1.25-0.93 (m, $7 \mathrm{H}), 0.83(\mathrm{t}, J=7.0 \mathrm{~Hz}, 3 \mathrm{H}) ;{ }^{13} \mathrm{C} \mathrm{NMR}\left(125 \mathrm{MHz}, \mathrm{CDCl}_{3}\right) \square 158.9,158.6$, 149.3, 135.8, 134.9, 127.6 ( 2 carbons), 127.5 (2 carbons), 113.6 (2 carbons), 113.3 (2 carbons), 112.8, 75.4, 55.25, 55.22, 51.9, 31.7, 29.5, 28.1, 27.4, 22.6, 14.1; IR (neat, $\mathrm{cm}^{-1}$ ) 3441 (br s), 1610 (s), 894 (m), 832 (s), 743 (w); HRMS (ES ) calcd for $\mathrm{C}_{24} \mathrm{H}_{36} \mathrm{NO}_{3}$ $\left(\mathrm{M}+\mathrm{NH}_{4}^{+}\right), 386.2695$, found 386.2686.

(ะ)- (1S, 2S)-(1-Cyclohexyl)-2-(1-hexyl)-3-(p-methoxyphenyl)-3-buten-1-ol (10a)

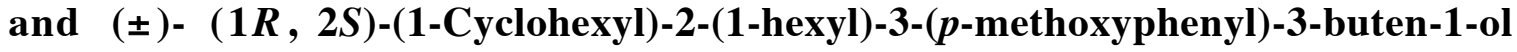
(10b). Treatment of cyclohexane carboxaldehyde $4 \mathbf{f}(0.025 \mathrm{~g}, 0.226 \mathrm{mmol}, 27 \square \mathrm{L}, 1.0$ equiv) according to the general procedure described above including the exact quantities of reagents, followed by flash chromatography over silica eluting with EtOAc/hexane (1 : 12), and preparative TLC eluting with EtOAc/hexane (1:12) afforded an inseparable mixture of diastereomers 10a and 10b $\left(0.036 \mathrm{~g}, 46 \%, \mathbf{1 0 a}: \mathbf{1 0 b}=3: 1,50 \%\right.$ de by $\left.{ }^{1} \mathrm{H} \mathrm{NMR}\right)$ as a colorless oil.

Analytical data for the mixture of diastereomers $10 \mathrm{a}: 10 \mathrm{~b}=\mathbf{3}: \mathbf{1}: \mathrm{R}_{f}=0.40$ (EtOAc/hexane $1: 5) ;{ }^{1} \mathrm{H}$ NMR (500 MHz, $\left.\mathrm{CDCl}_{3}\right) \square 7.29(\mathrm{~d}, J=8.3 \mathrm{~Hz}, 0.5 \mathrm{H}), 7.27$ (d, $J$ $=8.6 \mathrm{~Hz}, 1.5 \mathrm{H}), 6.86(\mathrm{~d}, J=8.8 \mathrm{~Hz}, 1.5 \mathrm{H}), 6.85(\mathrm{~d}, J=8.8 \mathrm{~Hz}, 0.5 \mathrm{H}), 5.37(\mathrm{~d}, J=1.0$ $\mathrm{Hz}, 0.75 \mathrm{H}), 5.34(\mathrm{~d}, J=0.6 \mathrm{~Hz}, 0.25 \mathrm{H}), 5.10(\mathrm{~d}, J=1.1 \mathrm{~Hz}, 0.25 \mathrm{H}), 5.03(\mathrm{~s}, 0.75 \mathrm{H})$, $3.82(\mathrm{~s}, 2.25 \mathrm{H}), 3.81$ (s, $0.75 \mathrm{H}), 3.36$ (dd, $J=12.2 \mathrm{~Hz}, 5.3 \mathrm{~Hz}, 0.25 \mathrm{H}), 3.11$ (pent, $J=3.8$ $\mathrm{Hz}, 0.75 \mathrm{H}), 2.77$ (dt, $J=11.3 \mathrm{~Hz}, 3.5 \mathrm{~Hz}, 1 \mathrm{H}), 1.86$ (s, br, $0.25 \mathrm{H}), 1.83$ (s, br, $0.75 \mathrm{H})$, 1.73-1.61 (m, 7 H), 1.50-1.42 (m, 3 H), 1.26-1.16 (m, 8 H), 1.13-1.05 (m, 2 H), 1.01-0.93 (m, $1 \mathrm{H}), 0.87(\mathrm{t}, J=6.8 \mathrm{~Hz}, 3 \mathrm{H}) ;{ }^{13} \mathrm{C}$ NMR (125 MHz, $\mathrm{CDCl}_{3}$ ) $\square$ 159.1, (158.9), (150.3), 
149.9, (136.5), 135.5, (127.9, 2 carbons), 127.7 (2 carbons), (113.8), 113.7 (2 carbons), (113.6, 2 carbons), 112.4, (78.3), 77.3, 55.3, (47.5), 46.4, (40.6), 39.9, (32.2), 31.8, (31.7), (30.7), 29.7, 29.6, (29.5), 28.9, 27.5, (27.4), 26.9, (26.5), 26.4, 26.3, (26.3), 25.9, 22.6, (22.6), 14.1, (14.0) signals for the minor diastereomer are given in parentheses; IR (neat, $\mathrm{cm}^{-1}$ ) 3471 (br m), 1608 (m), 835 (m), 738 (s); HRMS (ES) calcd for $\mathrm{C}_{23} \mathrm{H}_{40} \mathrm{NO}_{2}$ $\left(\mathrm{M}+\mathrm{NH}_{4}^{+}\right), 362.3059$, found 362.3067 .

(+)-5,6-Dihydro-4-(p-methoxyphenyl)-6-phenyl-2H-pyran-2-one (12). To a solution of $p$-methoxyphenylboronic acid $2 \mathbf{b}(0.069 \mathrm{~g}, 0.451 \mathrm{mmol}, 2.0$ equiv), (+)-(3,2,10- $\square-$ pinene)palladium(II) chloride $1 \mathbf{b}(0.013 \mathrm{~g}, 0.023 \mathrm{mmol}$. 0.1 equiv), tricyclohexylphosphine $\left(\mathrm{PCy}_{3}\right)(0.013 \mathrm{~g}, 0.045 \mathrm{mmol}, 0.2$ equiv) and $\mathrm{CsF}(0.137 \mathrm{~g}, 0.905 \mathrm{mmol}, 4.0$ equiv) in THF $(5.0 \mathrm{~mL})$ was injected neat ethyl 2,3-butadienoate $11(0.127 \mathrm{~g}, 1.13 \mathrm{mmol}, 131 \mathrm{LL}, 5.0$ equiv) and benzaldehyde $4 \mathbf{a}(0.024 \mathrm{~g}, 0.226 \mathrm{mmol}, 23 \mathrm{LL}, 1.0$ equiv). The reaction mixture was stirred at $\mathrm{rt}$ under argon for $24 \mathrm{~h}$. Water $(20 \mathrm{~mL})$ was added, and the mixture was extracted with ether $(4 \times 20 \mathrm{~mL})$. The organic extract was dried $\left(\mathrm{MgSO}_{4}\right)$, and the solvents were removed under reduced pressure to afford a crude product that was separated by flash chromatography over silica eluting with EtOAc/hexane (1:3). Subsequent purification via preparative thin layer chromatography over silica eluting with EtOAc/hexane (1:3) afforded lactone $12(0.041 \mathrm{~g}, 65 \%)$ as a white solid in $22.5 \%$ ee (by HPLC); [ $]_{\mathrm{D}}+37.8(\mathrm{c} 1.20$, $\left.\mathrm{CH}_{2} \mathrm{Cl}_{2}\right)$ : mp 120-122 ${ }^{\circ} \mathrm{C}($ EtOAc/hexane $3: 4) ; \mathrm{R}_{f}=0.47($ EtOAc/hexane $3: 4) ;{ }^{1} \mathrm{H}$ NMR $\left(500 \mathrm{MHz}, \mathrm{CDCl}_{3}\right) \square 7.53(\mathrm{~d}, J=8.9 \mathrm{~Hz}, 2 \mathrm{H}), 7.48(\mathrm{~d}, J=8.7 \mathrm{~Hz}, 2 \mathrm{H}), 7.42(\mathrm{t}, J=6.9 \mathrm{H}$, $2 \mathrm{H}), 7.40-7.36(\mathrm{~m}, 1 \mathrm{H}), 6.95(\mathrm{~d}, J=8.9 \mathrm{~Hz}, 2 \mathrm{H}), 6.40(\mathrm{~d}, J=1.6 \mathrm{~Hz}, 1 \mathrm{H}), 5.52(\mathrm{dd}, J=$ 10.2 Hz, $5.5 \mathrm{~Hz}, 1 \mathrm{H}), 3.85$ (s, $3 \mathrm{H}), 3.01-2.94$ (m, $2 \mathrm{H}) ;{ }^{13} \mathrm{C}$ NMR (125 MHz, $\left.\mathrm{CDCl}_{3}\right) \square$ 165.9, 161.9, 154.2, 138.9, 128.9 (2 carbons), 128.8, 128.2, 127.9 (2 carbons), 126.3 (2 carbons), 114.6 (2 carbons), 112.9, 78.9, 55.7, 34.3; IR (KBr, cm $\left.{ }^{-1}\right) 1704$ (s); HRMS (ES $\left.{ }^{+}\right)$ calcd for $\mathrm{C}_{18} \mathrm{H}_{17} \mathrm{O}_{3}\left(\mathrm{M}+\mathrm{H}^{+}\right), 281.1178$, found 281.1160 . 
Procedure for analyses of enantiomeric excess of homoallylic alcohol 5a and lactone 12 via chiral phase HPLC.

HPLC was performed on a Shimadzu LC-10Avp HPLC spectrometer with a CHIRALPAK AD or CHIRALCEL OD column at room temperature using a Shimadzu SPD-10Avp UV detector (210 or $245 \mathrm{~nm}$ ). Samples for HPLC analyses were prepared by dissolving 1-3 mg of the pure compounds in 2.0-6.0 mL of 2-propanol (HPLC grade). A microliter (1 $\square \mathrm{L}$ ) of the solution was injected for the HPLC analysis. HPLC grade 2-propanol, and hexane were used for the elution. The analytical conditions are listed in the following table.

Table S1. Determination of \%ee by HPLC

\begin{tabular}{|c|c|c|c|c|c|c|}
\hline entry & compound & column $^{a}$ & eluent & $\begin{array}{c}\text { flow rate } \\
(\mathrm{ml} / \mathrm{min})\end{array}$ & $\begin{array}{c}\text { retention time }^{b} \\
\text { of }(+) \text { isomer }\end{array}$ & $\begin{array}{c}\text { retention time }^{b} \\
\text { of }(-) \text { isomer }\end{array}$ \\
\hline 1 & $\mathbf{5 a}^{c}(0 \%$ ee $)$ & $\mathrm{A}$ & $10 \%^{d}$ & 1.0 & 10.1 & 7.84 \\
\hline 2 & $\mathbf{1 2}^{e}(23 \%$ ee $)$ & $\mathrm{B}$ & $15 \%^{d}$ & 1.0 & 50.6 & 42.4 \\
\hline
\end{tabular}

${ }^{a} \mathrm{~A}=$ CHIRALPAK AD, B = CHIRALCEL OD. ${ }^{b}$ in minutes. ${ }^{c}$ Detection at 245 nm. ${ }^{d} \%$ of 2-propanol in hexane. ${ }^{e}$ Detection at $210 \mathrm{~nm}$.

\title{
References.
}

1 Buchman, O.; Grosjean, M.; Nasielski, J. Bull. Soc. Chem. Belges. 1962, 71, 467472.

2 Moreaou, J.-L.; Gaudemar, M. J. Organomet. Chem. 1976, 108, 159-164.

3 Hosokawa, T.; Uno, T.; Inui, S.; Murahashi, S.-I. J. Am. Chem. Soc. 1981, 103, 2318-2323.

\author{
NOTE: IN THE FOLLOWING ${ }^{1} \mathrm{H}$ NMR AND ${ }^{13} \mathrm{C}$ NMR SPECTRA, \\ OCCASIONALLY PRESENT WEAK SIGNALS AT: \\ ${ }^{1} \mathrm{H}$ NMR: $1.50 \mathrm{ppm}\left(\mathrm{H}_{2} \mathrm{O}\right), 2.05 \mathrm{ppm}$ (acetone), $5.32 \mathrm{ppm}\left(\mathrm{CH}_{2} \mathrm{Cl}_{2}\right)$ \\ ${ }^{13}$ C NMR: 29.9 ppm (acetone)
}

CORRESPOND TO THE RESIDUAL SOLVENTS LISTED IN PARENTHESES. 


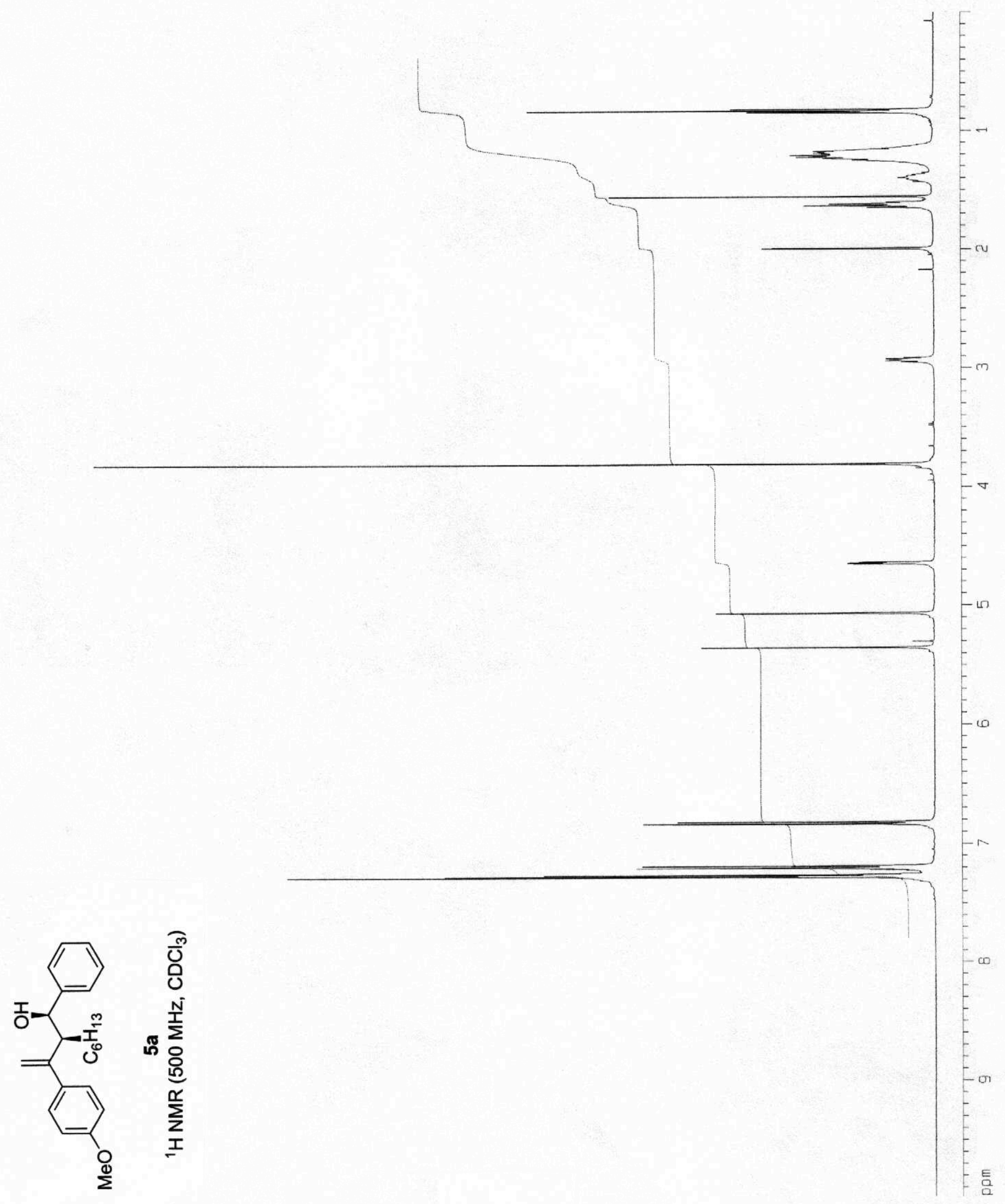




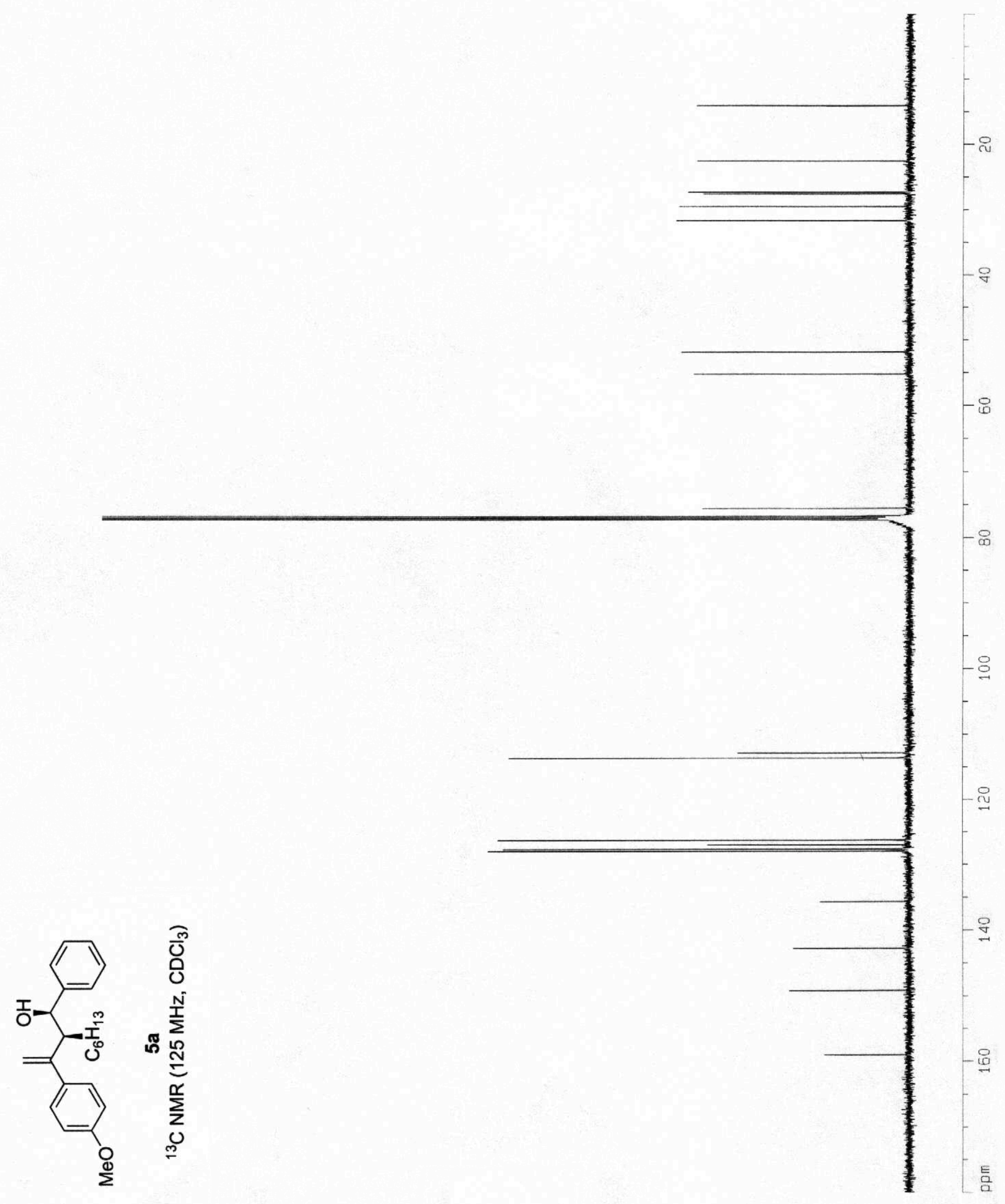

S-12 


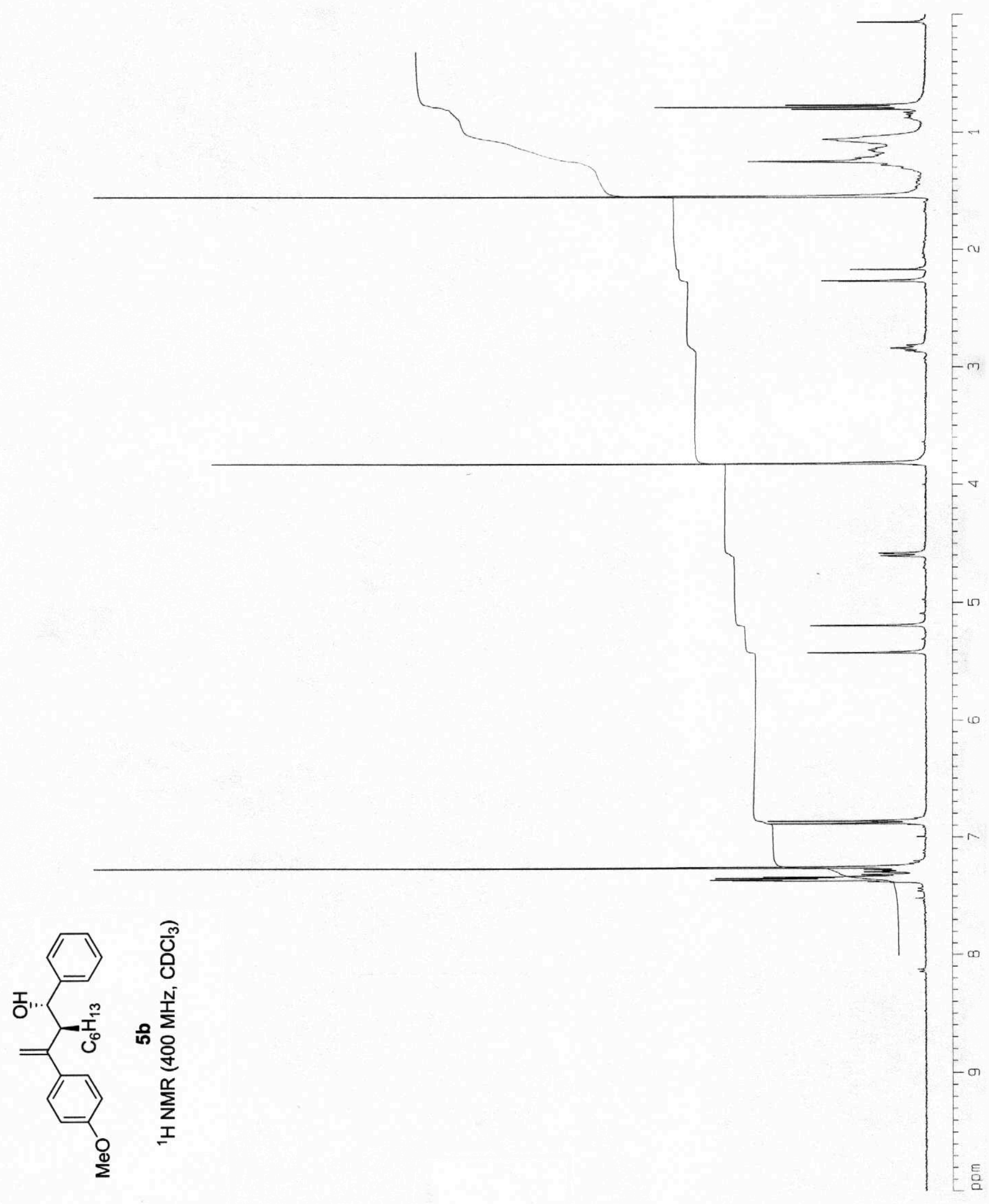




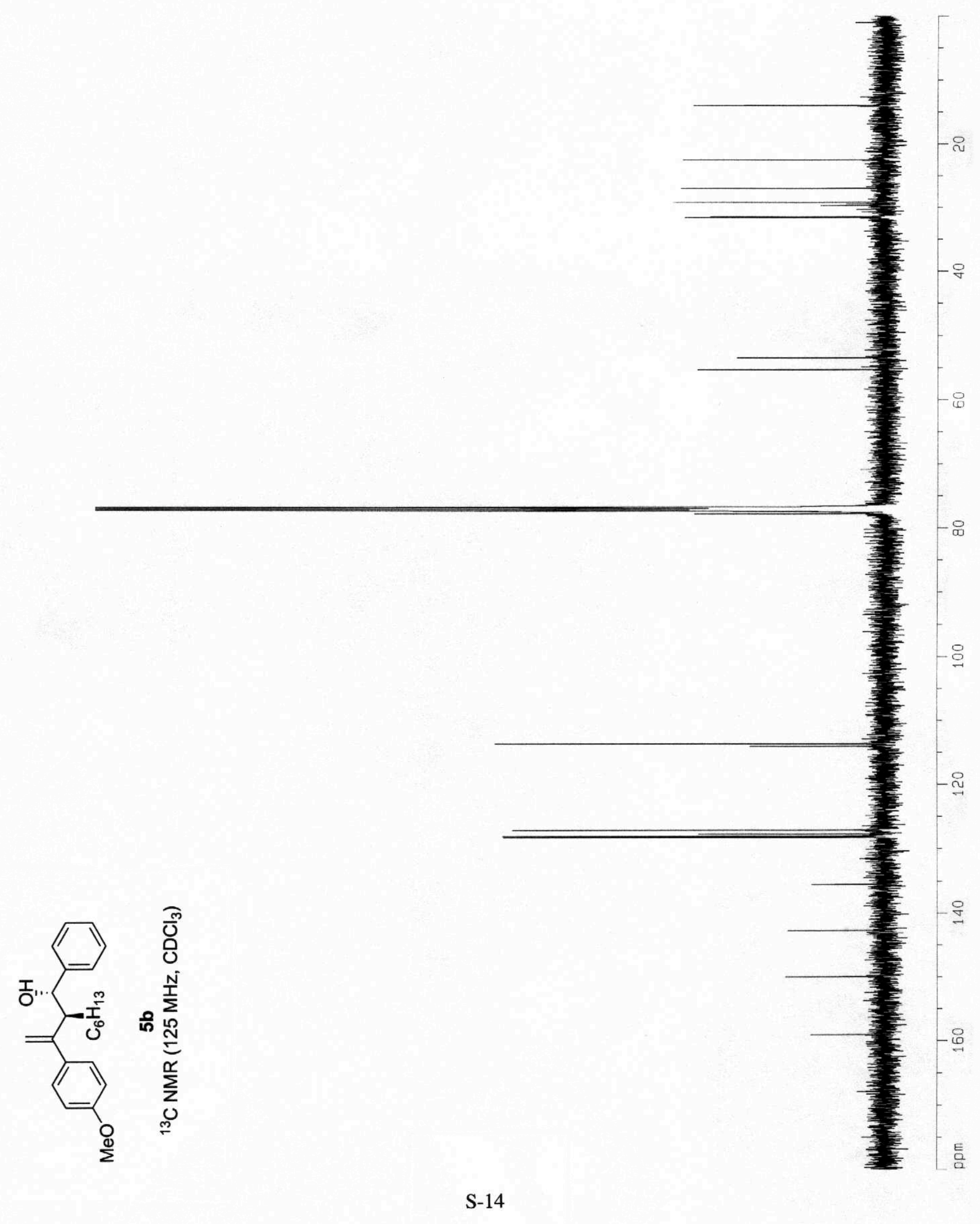




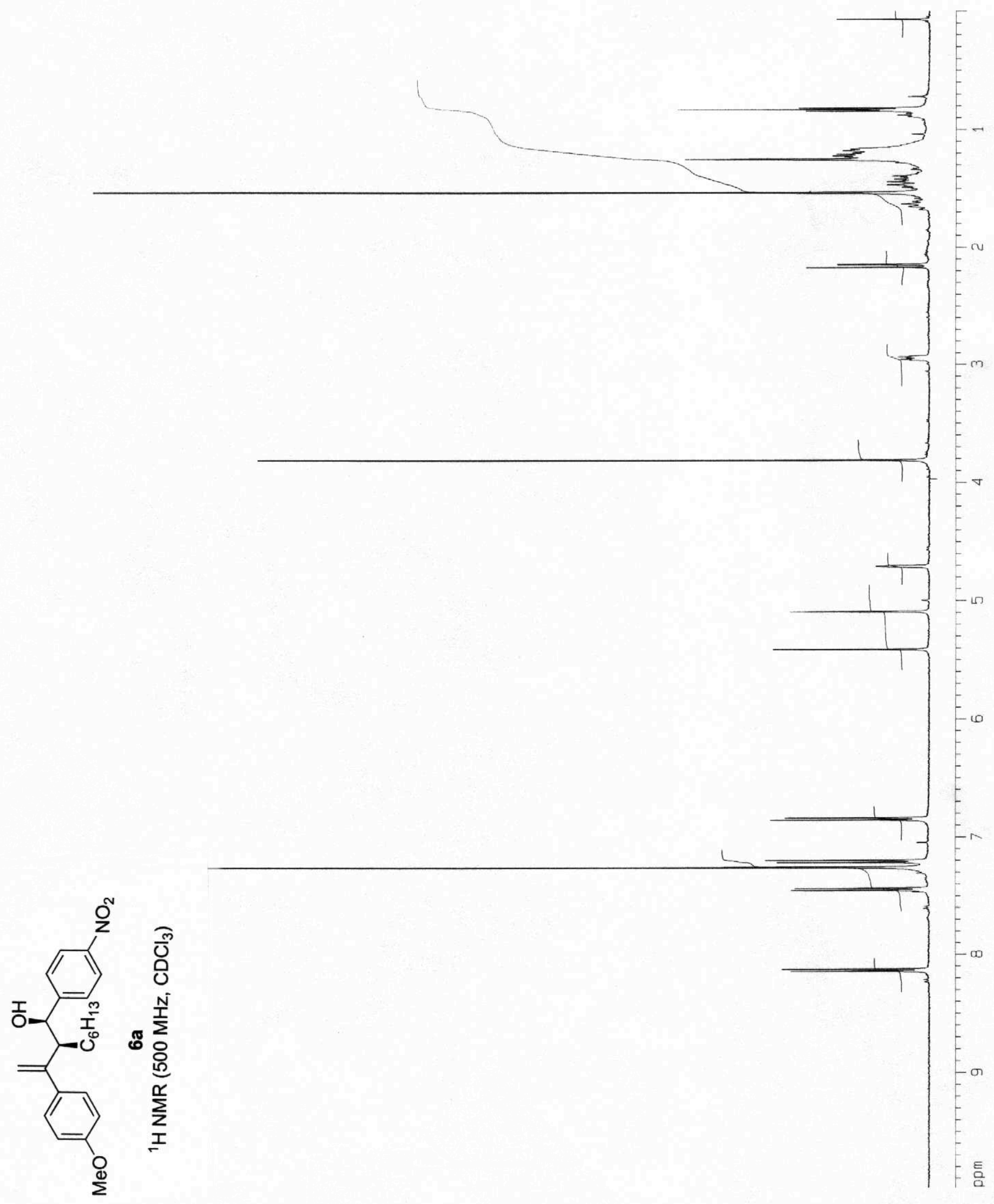




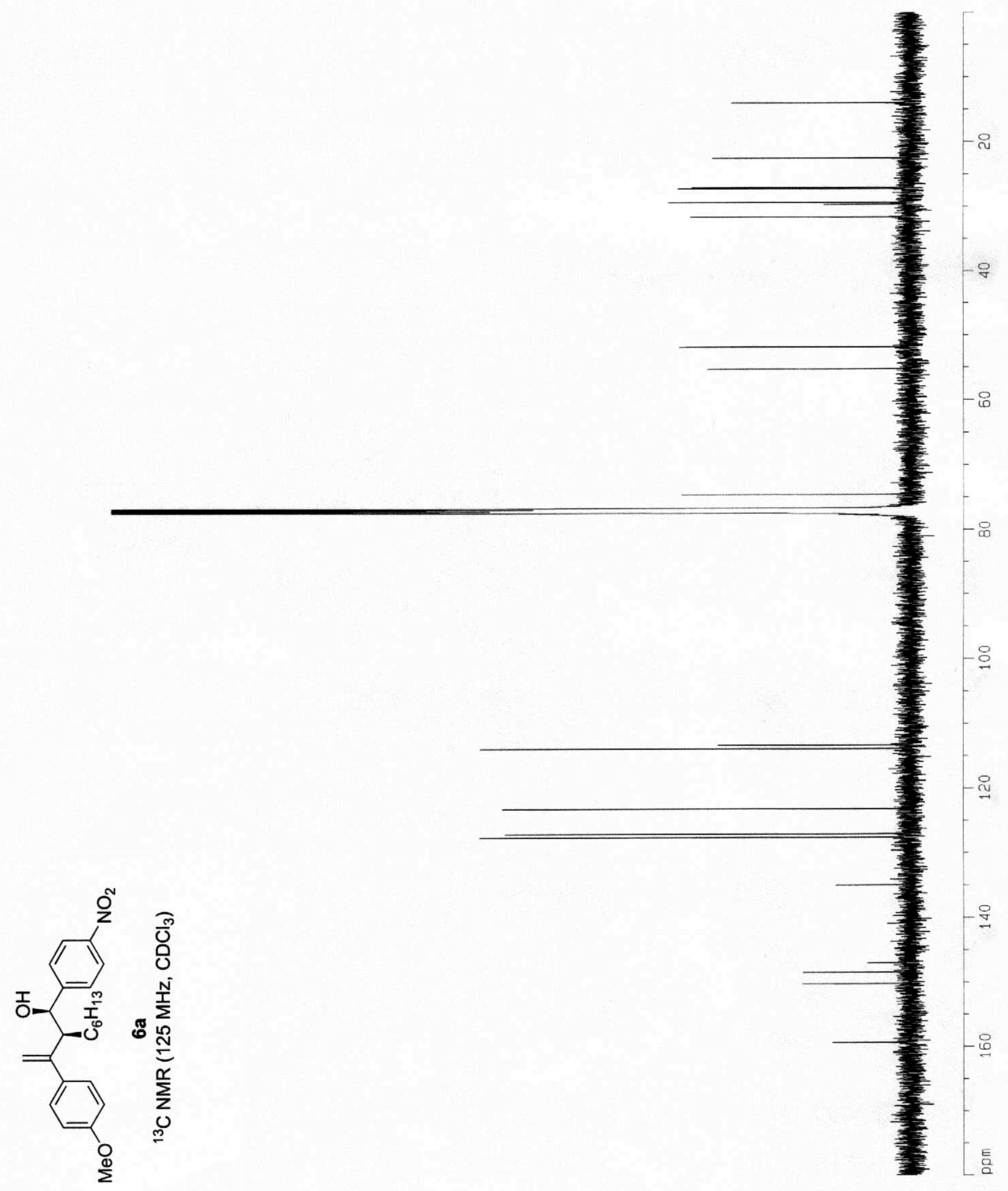




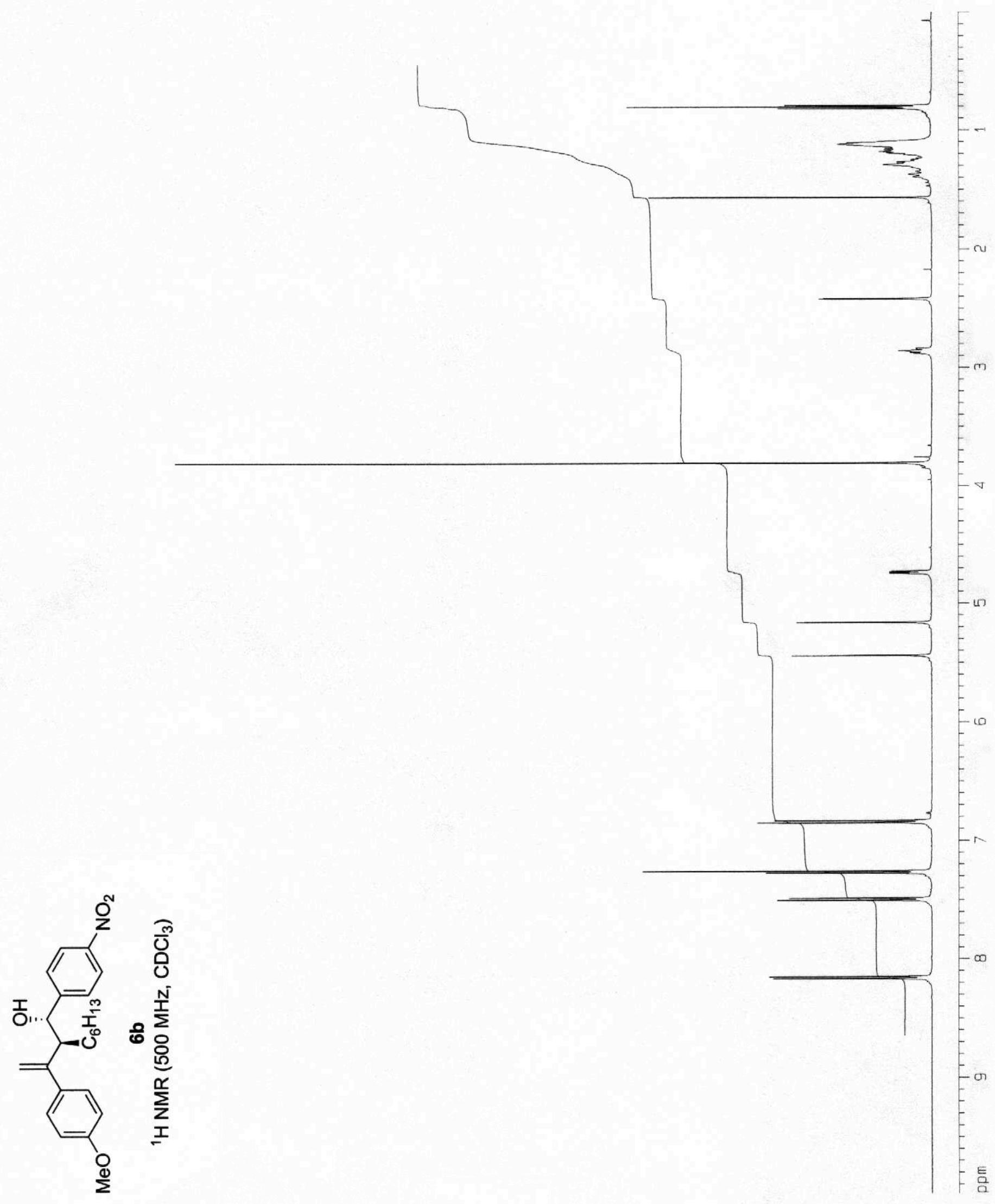




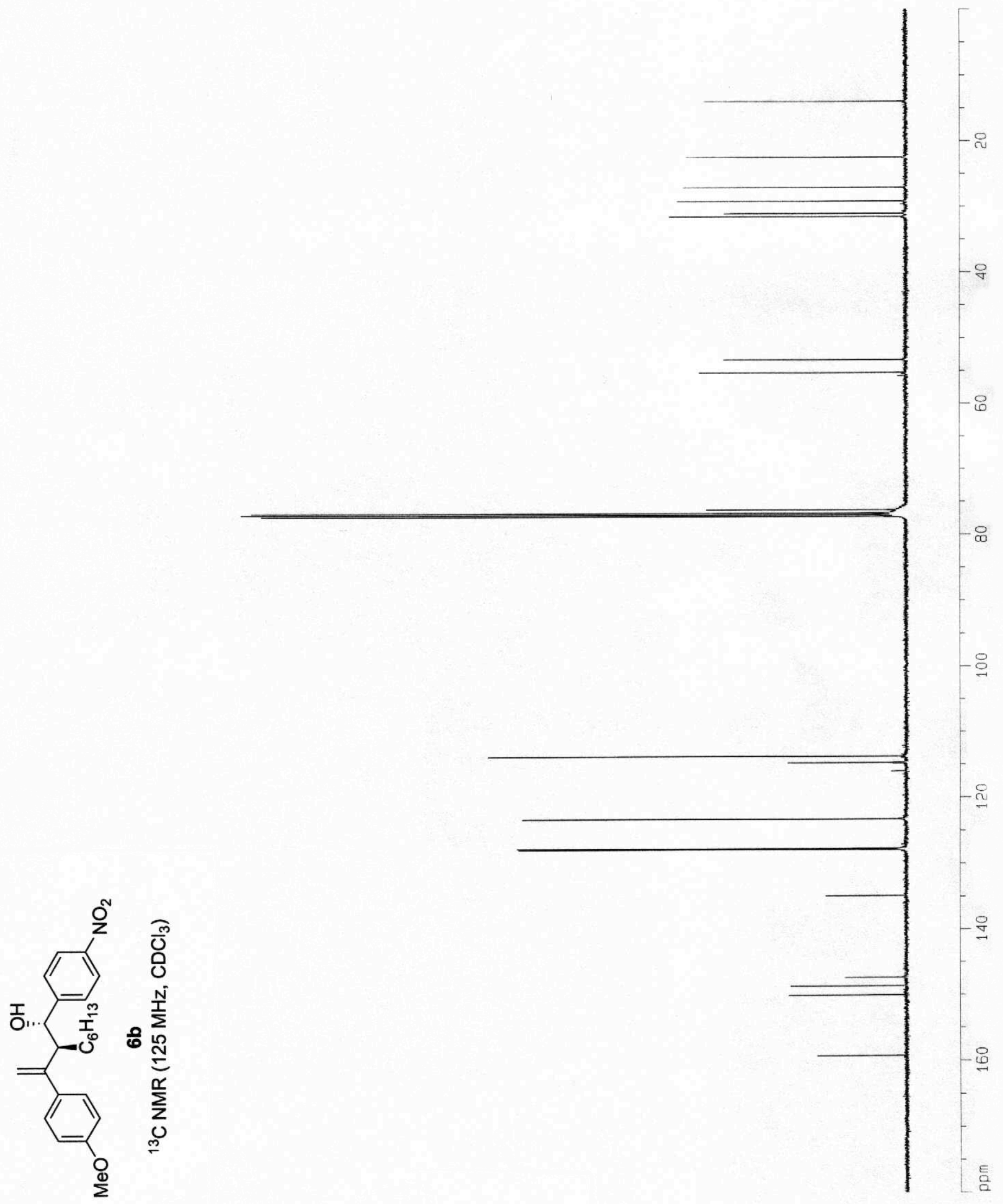




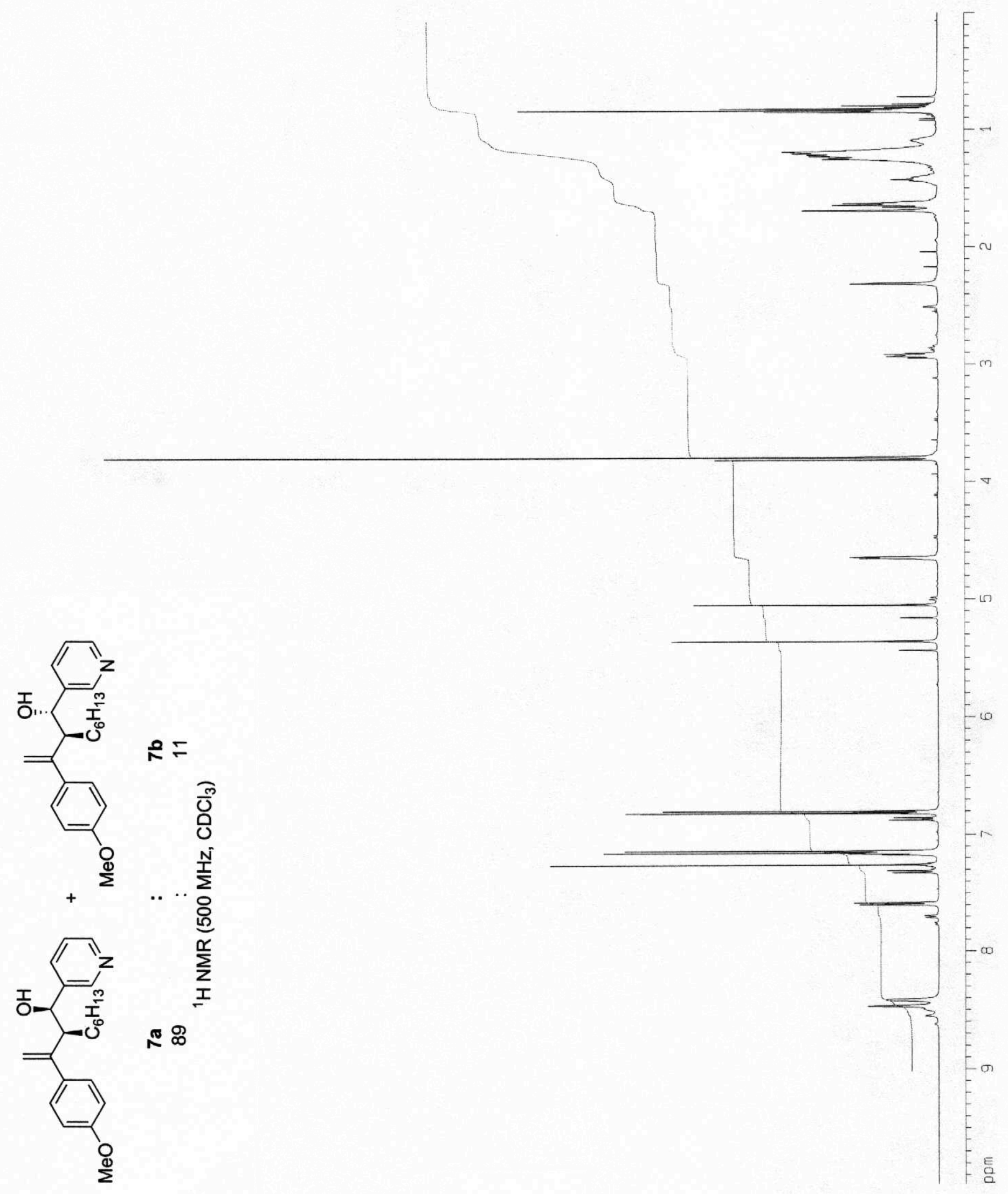




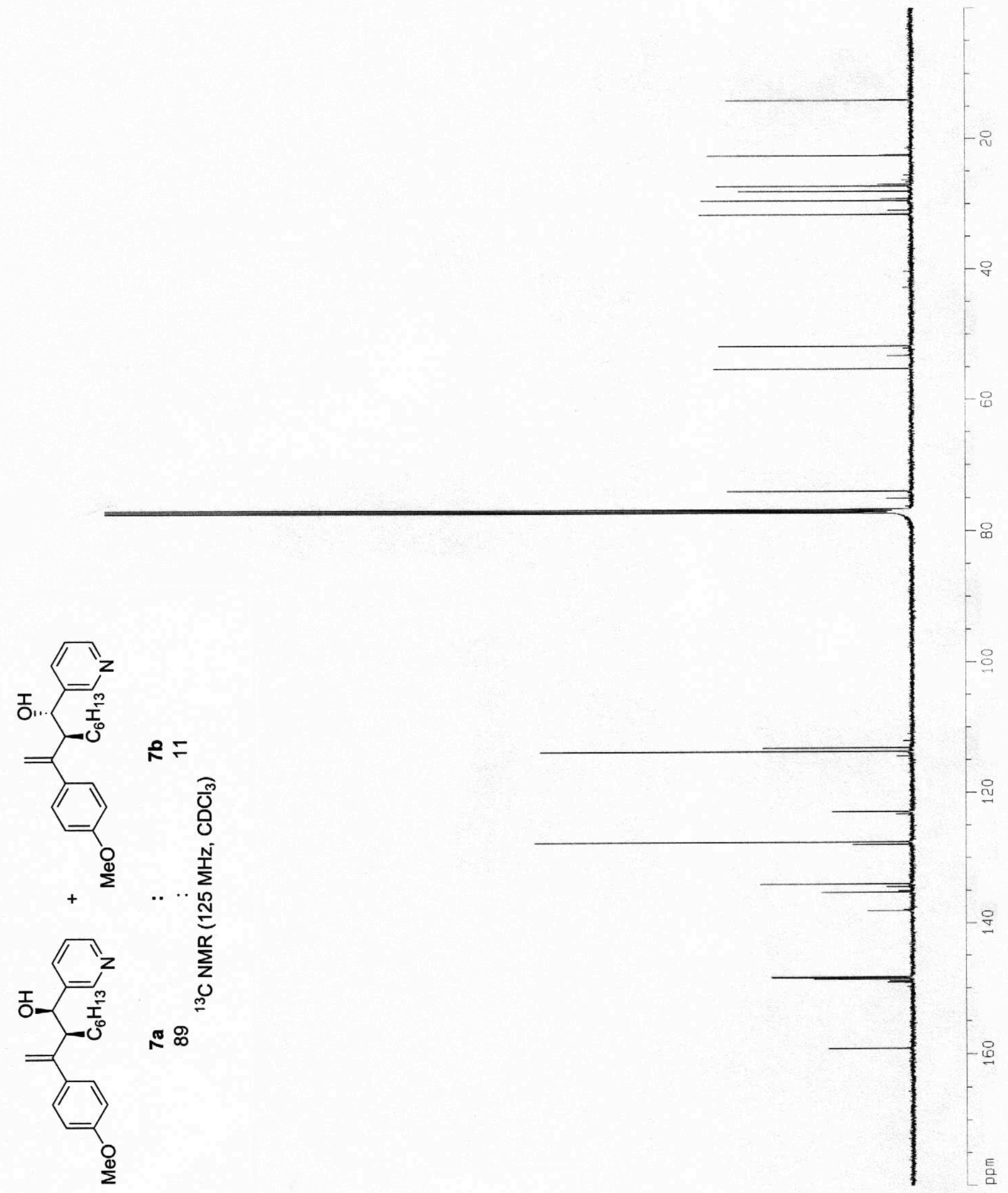




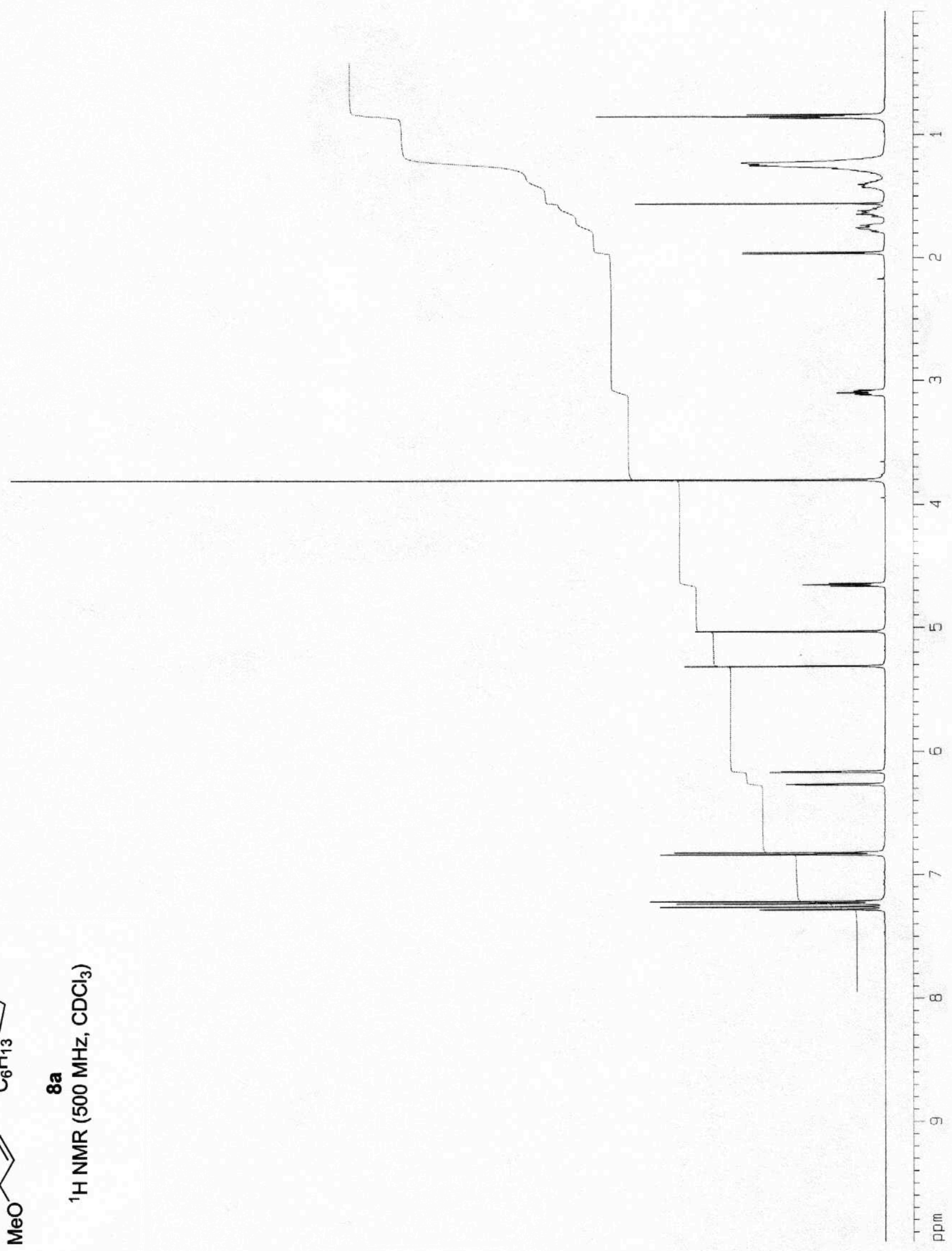




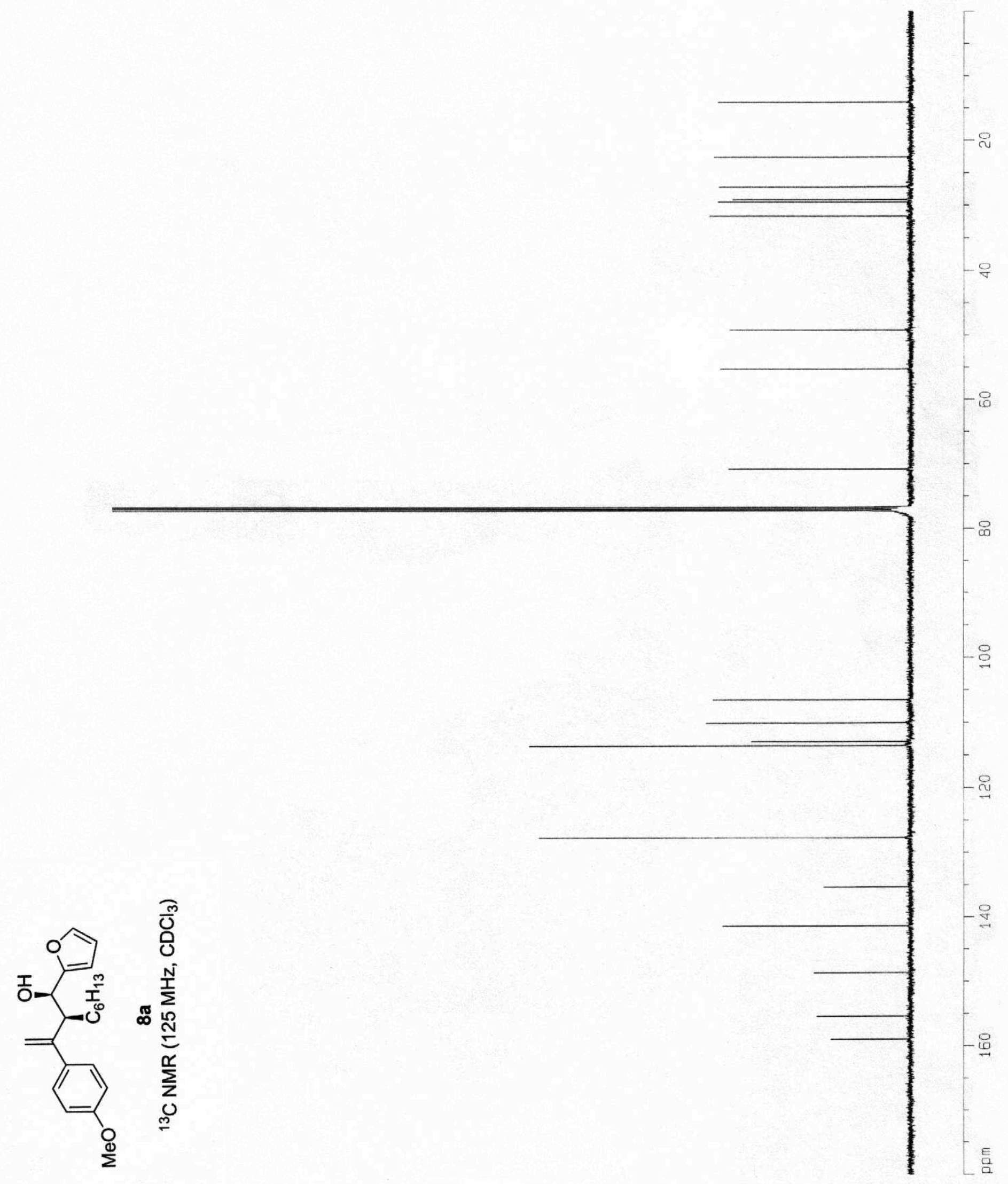




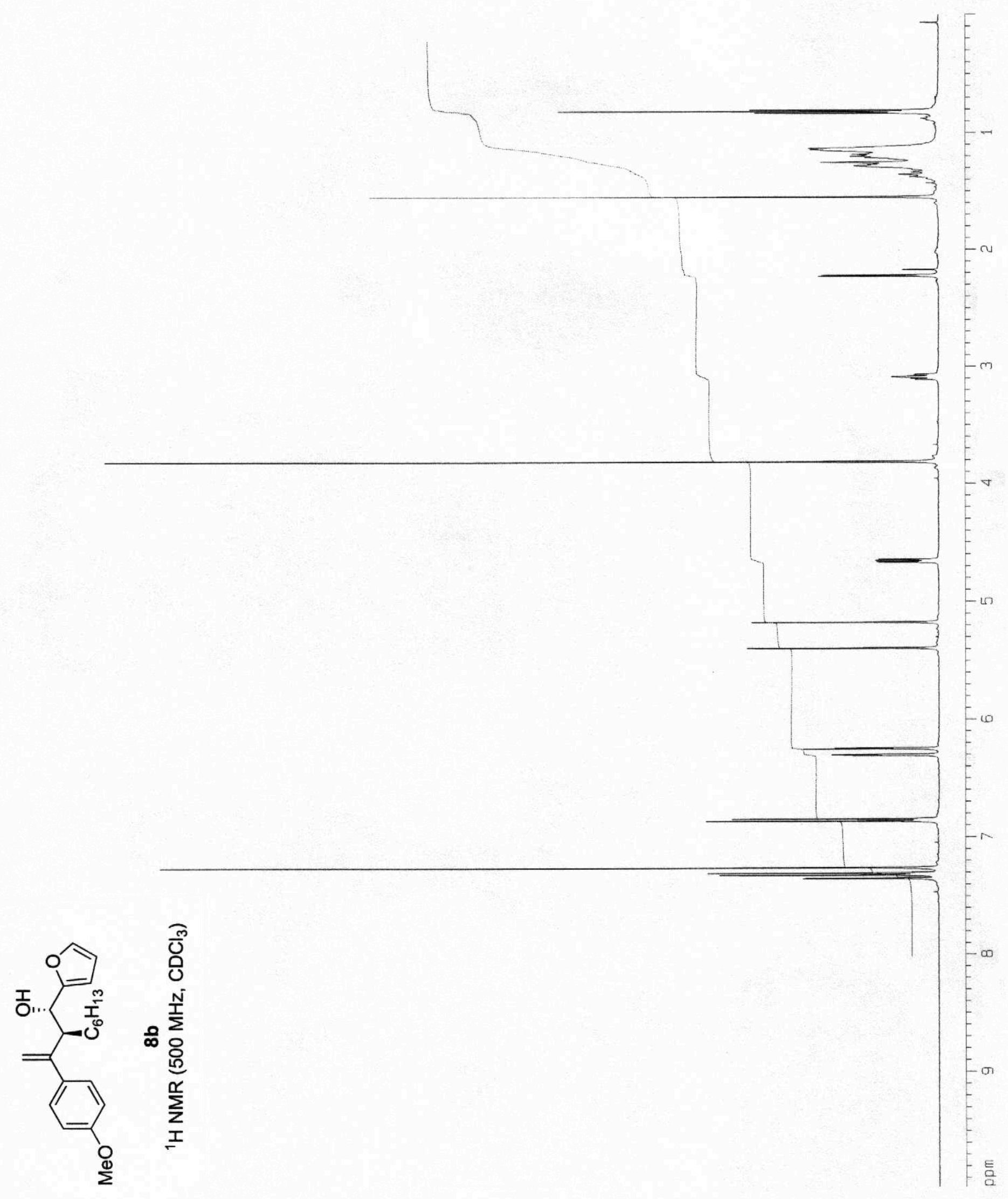




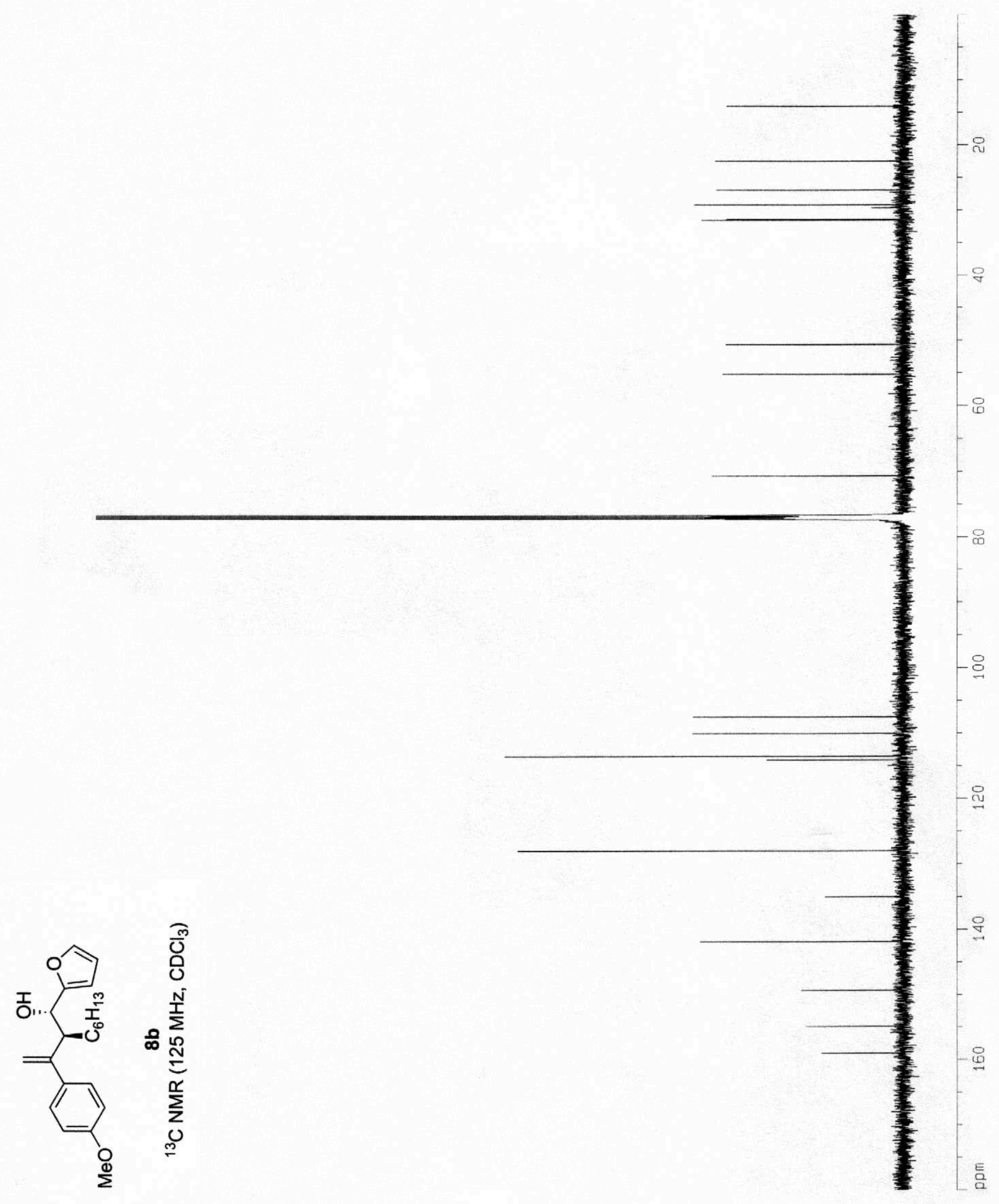




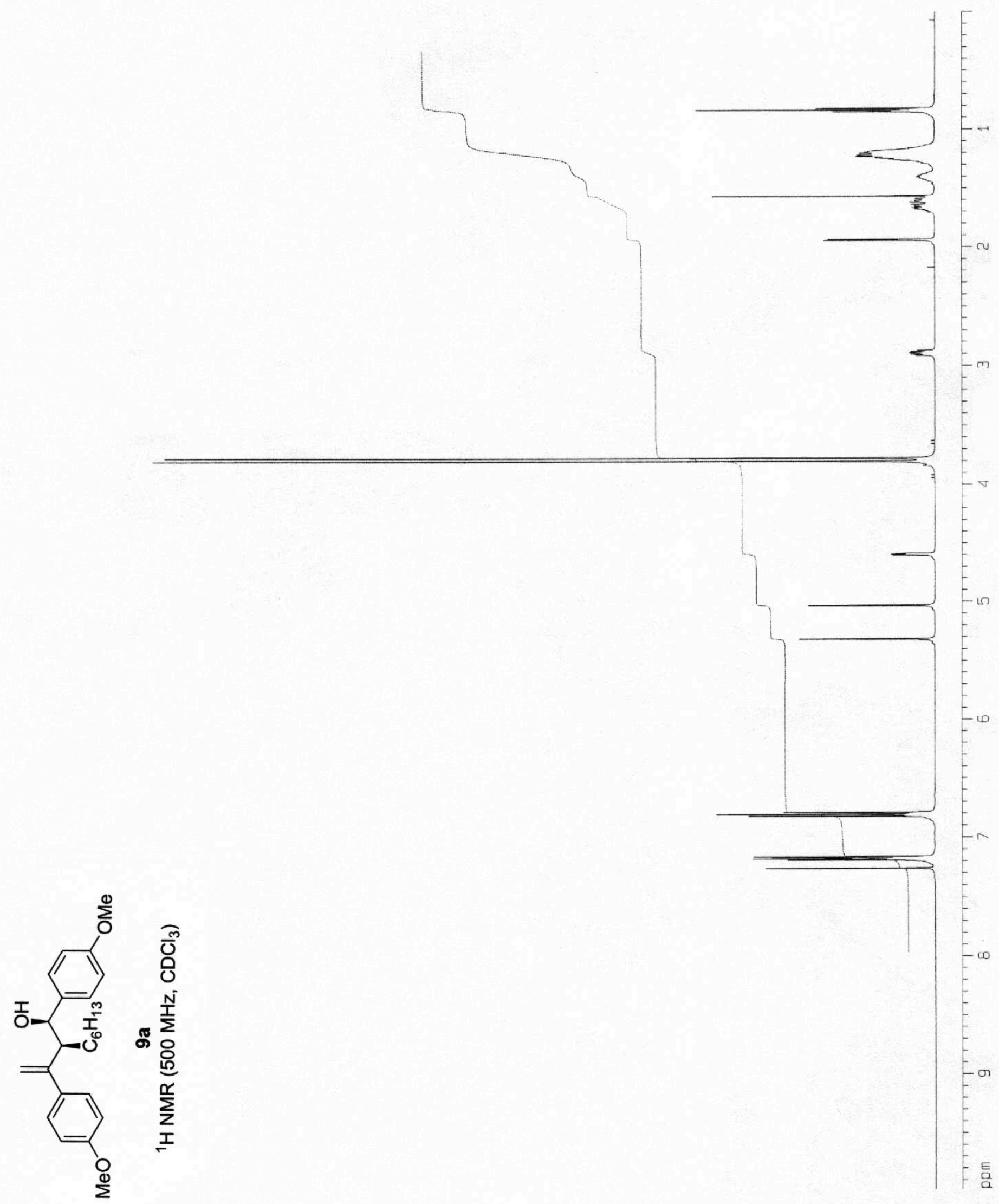




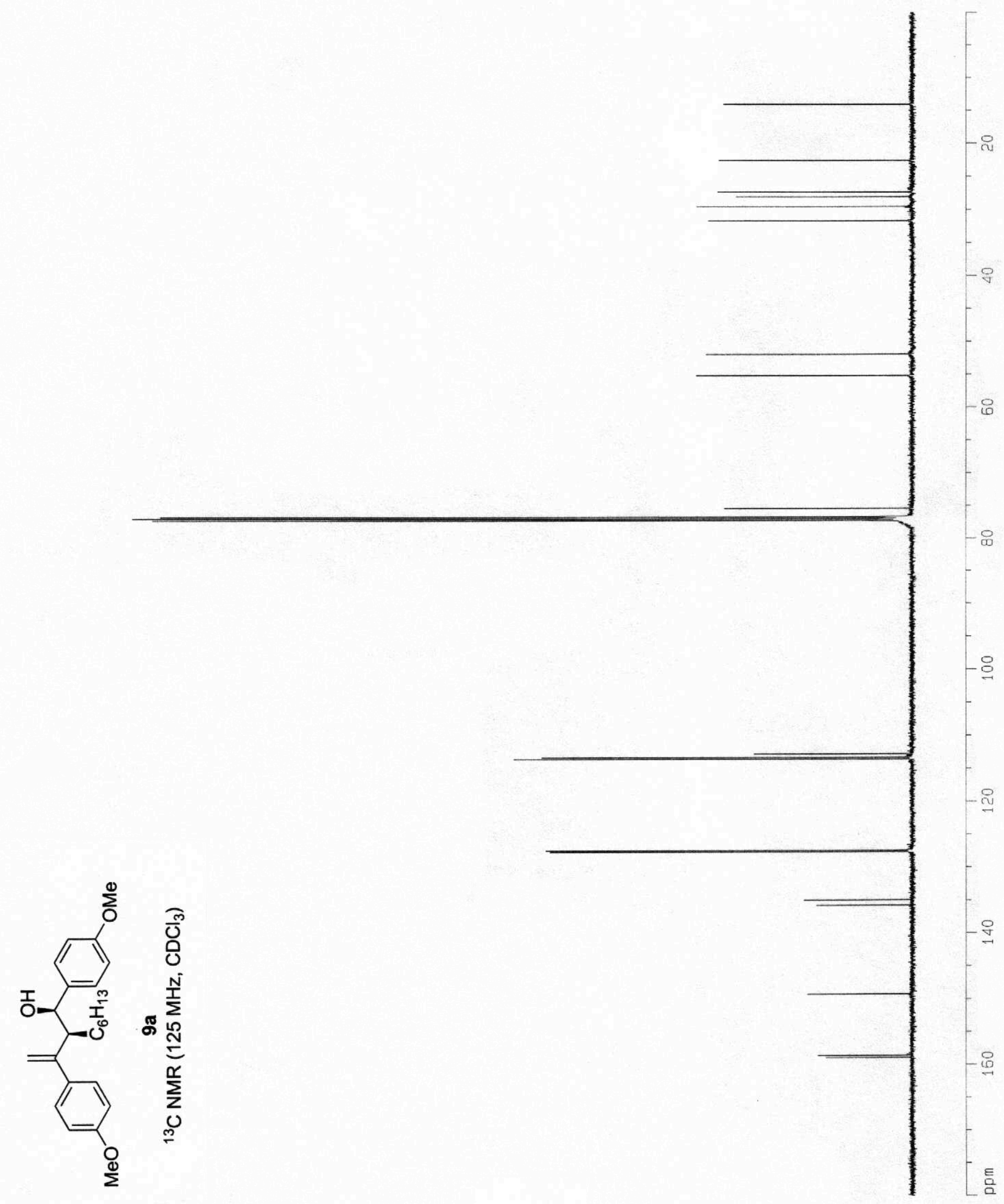




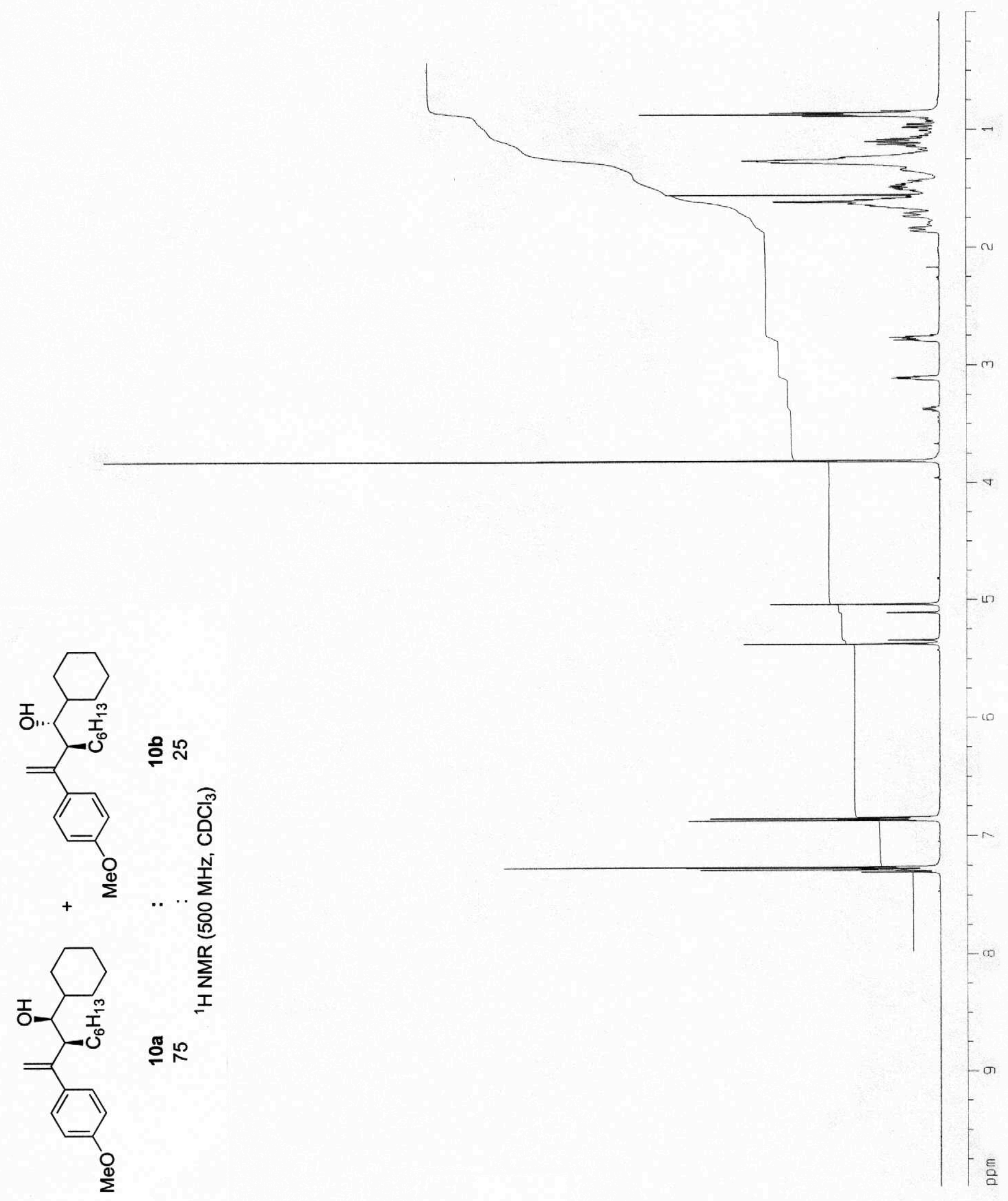




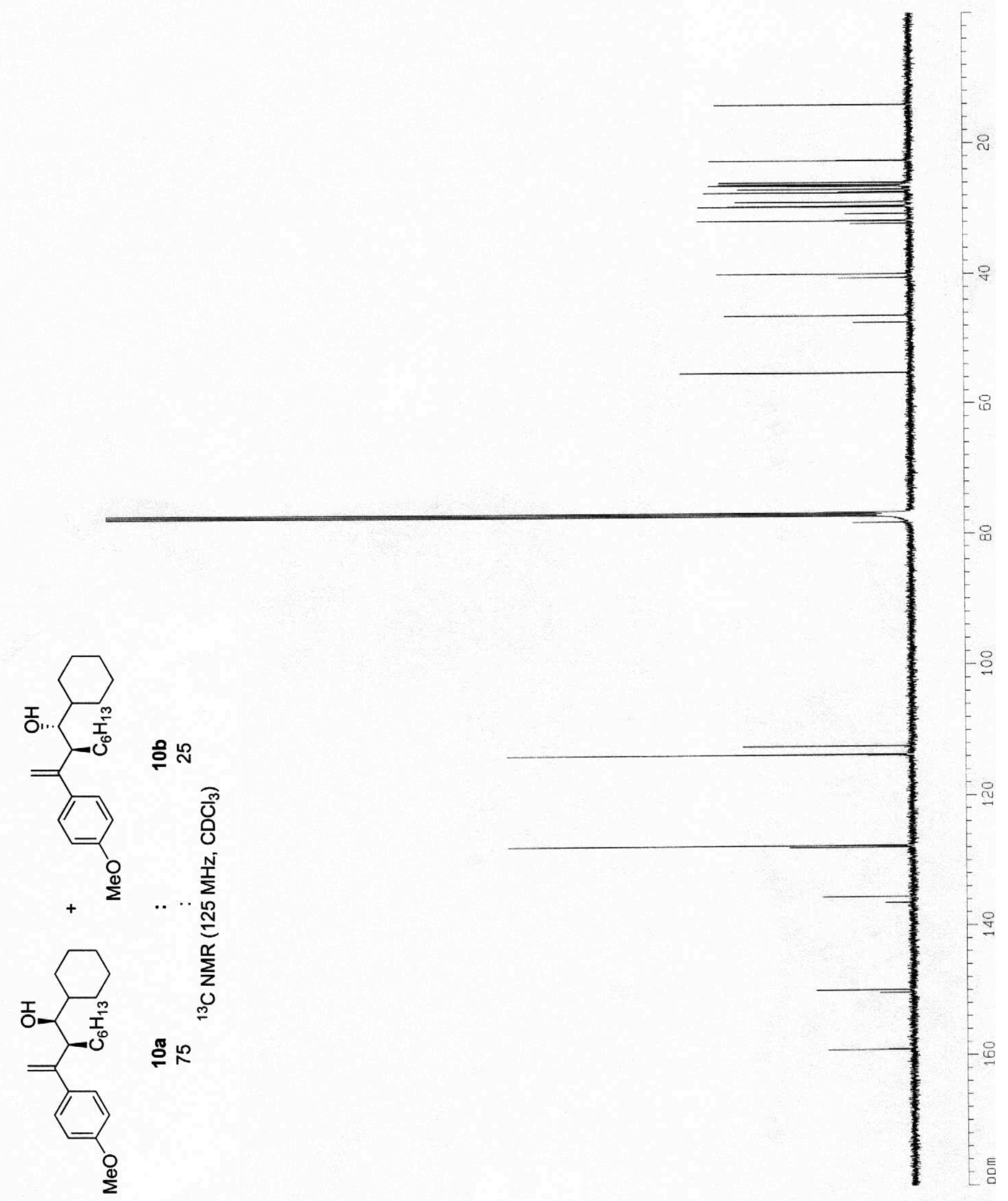




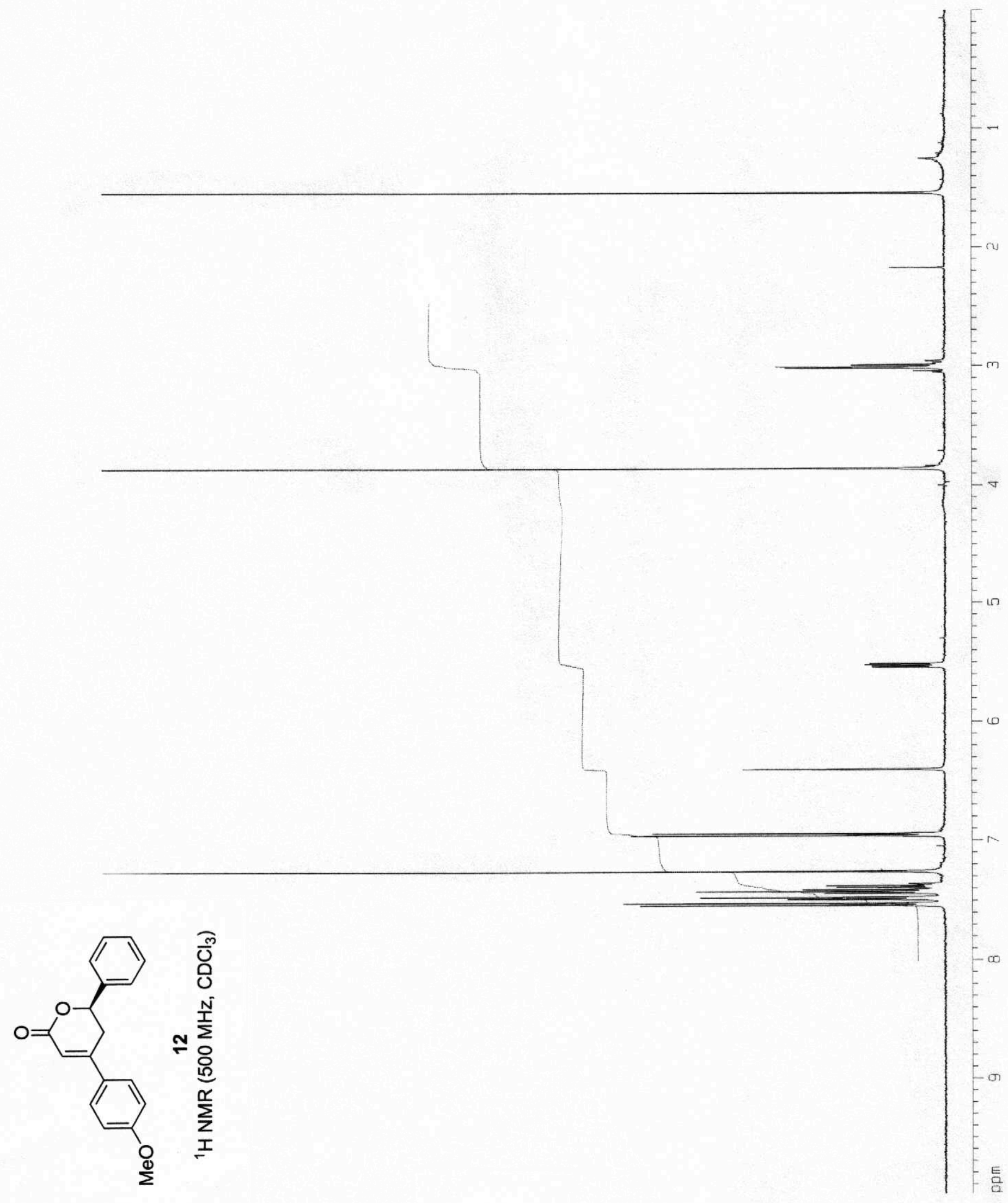




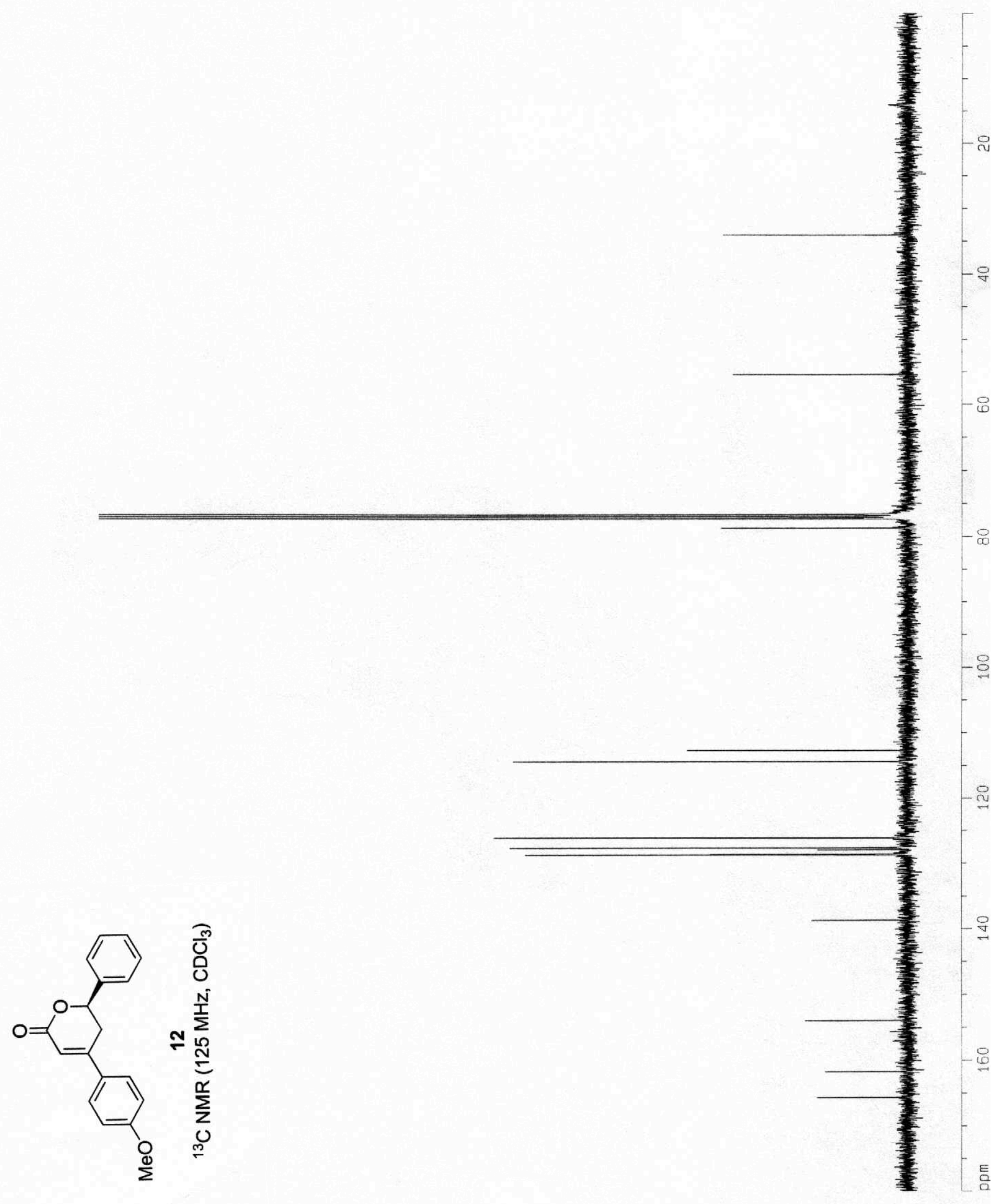

